\title{
OBSTRUCTIONS TO TRIVIALIZING A KNOT
}

\author{
Joan S. Birman and John Atwell Moody \\ submitted June 11, 2002; revised October 16, 2003
}

\begin{abstract}
The recent proof by Bigelow and Krammer that the braid groups are linear opens the possibility of applications to the study of knots and links. It was proved by the first author and Menasco that any closed braid representative of the unknot can be systematically simplified to a round planar circle by a finite sequence of exchange moves and reducing moves. In this paper we establish connections between the faithfulness of the KrammerLawrence representation and the problem of recognizing when the conjugacy class of a closed braid admits an exchange move or a reducing move.
\end{abstract}

\section{Introduction}

The goal of this article is to study some problems in algorithmic knot theory with the use of new tools. Our work will combine two threads of thought:

- The first author's work with Menasco on the study of knots via closed braids.

- The work of the second author, who proved that the faithfulness of certain matrix representations of the braid group rested on whether the matrices in question effectively detected intersections between certain arcs on the punctured plane and their images under braid homeomorphisms.

Let $\mathcal{K}$ be an oriented knot type in oriented 3 -space $S^{3}$, or alternatively in $\mathbb{R}^{3}$. A representative $\tilde{K} \in \mathcal{K}$ is said to be a closed braid if there is an unknotted curve A (the braid axis), which we shall think of as the z-axis in $\mathbb{R}^{3}$, and a choice of fibration of $\mathbb{R}^{3}-\mathbf{A}$ by half-planes $\{P \times\{t\}, t \in[0,1]\}$, such that whenever $\tilde{K}$ meets a fiber $P \times\{t\}$ the intersection is transverse. This implies that $\tilde{K} \subset\left(\mathbb{R}^{3}-\mathbf{A}\right)$. The braid index $n=n(\tilde{K})$ is the number of points in $\tilde{K} \cap(P \times\{t\})$. This number is independent of the choice of $t \in[0,1]$, because $\tilde{K}$ is transverse to every fiber $P \times\{t\}$. Two closed braids $\tilde{K}, \tilde{K}^{\prime} \subset\left(\mathbb{R}^{3}-\mathbf{A}\right)$ are equivalent if there is is an isotopy $\varphi:\left(\mathbb{R}^{3}-\mathbf{A}\right) \times[0,1] \rightarrow\left(\mathbb{R}^{3}-\mathbf{A}\right)$ with $\varphi(\tilde{K}, 0)=\tilde{K}$ and $\varphi(\tilde{K}, 1)=\tilde{K}^{\prime}$ and each $\varphi(\tilde{K}, s)$ a closed braid. Cutting $\mathbb{R}^{3}-\mathbf{A}$ along any half-plane $P \times\left\{t_{0}\right\}$ we obtain a braid $K$. Two open braids obtained by cutting along the same half-plane $P \times\left\{t_{0}\right\}$ are equivalent if there is an isotopy $\varphi$ as above which is the identity on the cutting plane.

Open braids are in 1-1 correspondence with elements in Artin's braid group $\mathbf{B}_{n}$ and closed braids are in 1-1 correspondence with conjugacy classes in $\mathbf{B}_{\mathbf{n}}$. The open braids obtained by cutting along distinct planes are conjugate in $\mathbf{B}_{n}$. 


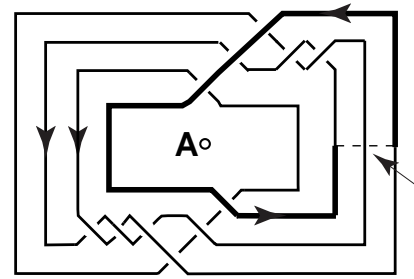

(a)

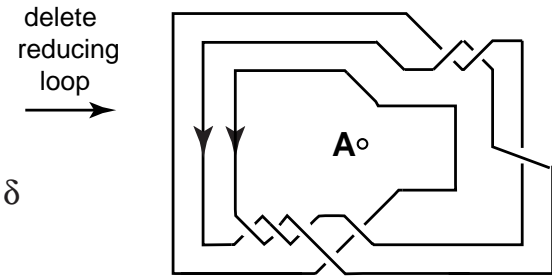

(b)

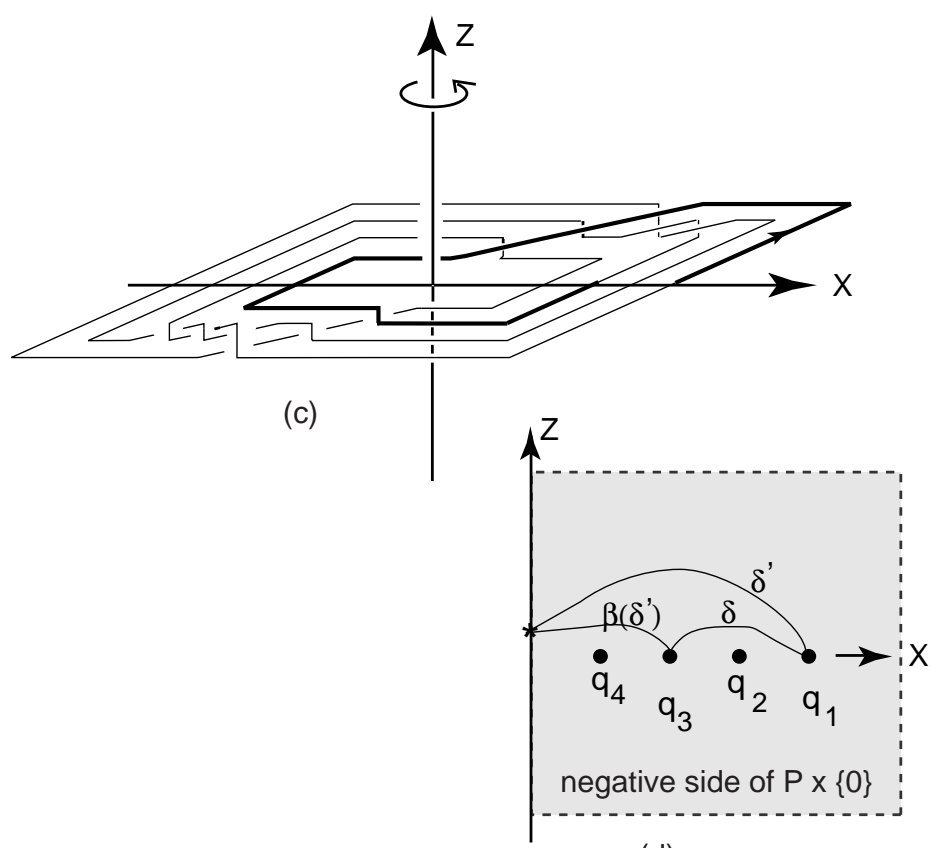

(d)

Figure 1: The closed 4-braid $\sigma_{2}^{-2} \sigma_{1}^{-1} \sigma_{2}^{-1} \sigma_{3}^{-1} \sigma_{2}^{3} \sigma_{1} \sigma_{2} \sigma_{3}$ has a reducing loop. 
We now define what we mean when we say that the conjugacy class of a closed braid $\tilde{K}$ 'admits a reducing move'. An example is given in Figure 11 The plane $P \times\{0\}$ meets $\tilde{K}$ in $n$ points $q_{1}, \ldots, q_{n}$. These $\mathrm{n}$ points divide $\tilde{K}$ into $n$ arcs $\alpha_{1}, \ldots, \alpha_{n}$, where each $\alpha_{i} \subset \tilde{K}$ begins at $q_{i}$ and ends at some $q_{\mu_{i}}$. Then $\tilde{K}$ admits a braid-index reducing move if for some $i \in\{1, \ldots, n\}$ there exists an arc $\delta$ in $P \times\{0\}$ which joins $q_{i}$ to $q_{\mu_{i}}$, such that the closed curve $\alpha_{i} \cup \delta_{i}$ bounds a disc $\Delta$ which intersects $\tilde{K}$ precisely in the braid strand $\alpha_{i}$. See sketches (a) and (d) of Figure 1 for an example. The caption describes the example in terms of the standard elementary braid generators $\sigma_{1}, \ldots, \sigma_{n-1}$ for $\mathbf{B}_{n}$.

If one deforms $\delta$ in $P \times\{0\}$ to $\delta^{\prime}$, where $\delta^{\prime}$ has a point on $\mathbf{A}$, as in sketch (d), then $\delta^{\prime}$ will sweep out the disc $\Delta$ as it is moved around the axis, keeping one endpoint fixed on $\mathbf{A}$ and sliding the other along $\tilde{K}$ to $\beta\left(\delta^{\prime}\right)$. Thus, if $\delta$ exists, then the closed $n$-braid $\tilde{K}$ can be replaced by a new representative of the same knot type, $\tilde{K}-\alpha_{i}+\delta$, by pushing $\alpha_{i}$ across $\Delta$ to $\delta$. Moreover, after tilting $\delta$ slightly to make it transverse to the fibration, the modified representative $\tilde{K}^{\prime}$ will be an $(n-1)$-braid representative of the same knot type. See sketch (b). Thus the closed $n$-braid $\tilde{K}$ is reducible to a closed $(n-1)$-braid, and so we say that it admits a reducing move or has a reducing loop. If we orient the braid strands anticlockwise, and number them along the positive $\mathrm{x}$ axis so that the one that is furthest from the braid axis $A$ is strand 1, then in the example $n=4, i=1$ and $\mu_{i}=3$. Remark: this numbering is correct because each $P \times\{t\}$ has a natural orientation, when we think of it as a disc in $S^{3}$, i.e. the orientation determined by the orientation on $\mathbf{A}=\partial(P \times\{t\})$. In Figure प(d) the $\mathrm{z}$-axis and the positive $\mathrm{x}$-axis determine the plane $P \times\{0\}$, and in sketch (d) we are seeing it from its negative side.

Observe that the property ' $K$ admits a reducing loop' is a property of the conjugacy class of $K$. For, the reducing arc $\alpha_{i}$ meets every fiber $P \times\{t\}, \quad t \in[0,1]$, so that we can find a reducing $\operatorname{arc} \alpha_{i}(t)$ by cutting $\tilde{K}$ along $P \times\{t\}$ if and only if we can find a reducing arc $\alpha_{i}=\alpha_{i}(0)$ by cutting along $P \times\{0\}$.

We next define the sign of a reducing loop. Assume that $\tilde{K}$ admits a reducing move at the $\operatorname{arc} \delta$, as in Figure 1(a) or 2 After deleting the reducing loop the algebraic crossing number of the braid, i.e. its exponent sum when described by a word in the standard generators of $\mathbf{B}_{n}$, will either decrease or increase by 1 . If it decreases (resp. increases) we say that the reducing loop was positive (resp. negative). The left and right examples in Figure 2 are positive and negative respectively. The reducing loop in Figure 1(a) is positive because the algebraic crossing number goes down by 1 after the reduction.

The conjugacy class $\tilde{K}$ is said to admit an exchange move if it has a representative $K$ which has the special form $P \sigma_{n-1} Q \sigma_{n-1}^{-1}$, where $P$ and $Q$ only use strands $1, \ldots, n-1$. See Figure 3 Note that if $K$ admits an exchange move, then $K$ is a product of two reducible braids, $P \sigma_{n-1}$ and $Q \sigma_{n-1}^{-1}$ of opposite sign.

The knot or link type $\mathcal{K}$ is said to be exchange reducible if, up to conjugacy, any closed braid representative can be reduced to any representative of minimum braid index by a sequence of reducing and exchange moves. 

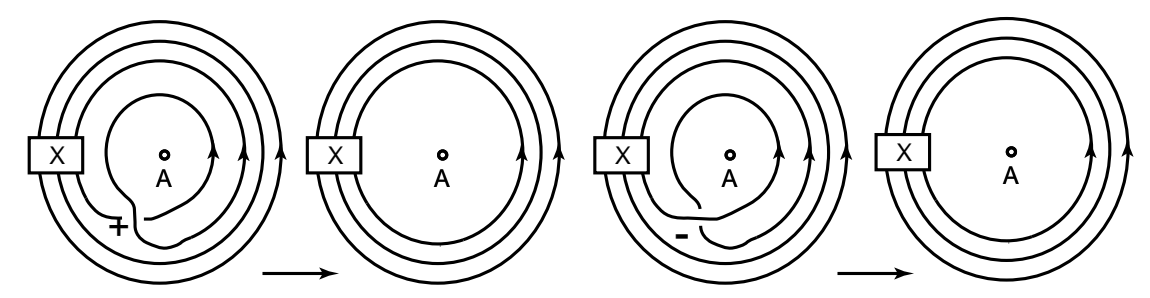

Figure 2: Positive and negative reducing loops

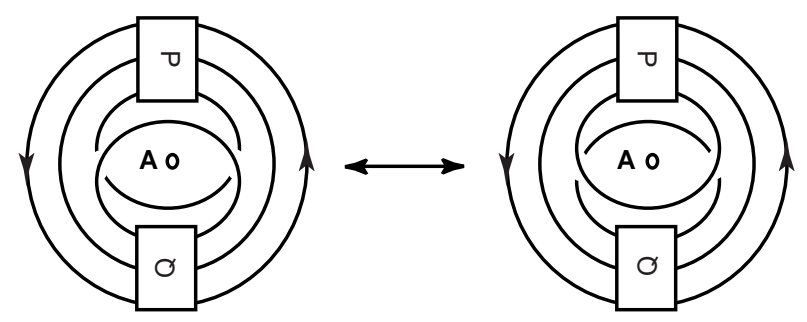

Figure 3: The exchange move

Here are some reasons why reducing and exchange moves are of interest in algorithmic knot theory:

1. In [3] it was shown that if $\mathcal{K}$ is a split or composite link type, then exchange moves alone suffice to modify any closed braid representative $\tilde{K}$ to a split or composite closed braid.

2. It was proved in [4] that the unlink on any number of components is exchange-reducible.

3. It was proved In 24 that iterated torus knots and links are exchange-reducible.

4. Exchange moves (together with isotopy in the complement of the braid axis) can lead to a major complication in the study of knots via closed braids, namely exchange moves result in infinitely many conjugacy classes of closed $n$-braid representatives of a knot or link, all related by exchange moves. See Figure [ Fortunately (see [5]) it has been proved that if a knot or link has infinitely many conjugacy classes of n-braid representatives then all but finitely many of them are related by exchange moves. Since it is fairly obvious from the pictures in Figure 4 that there ought to be a class of 'minimum complexity', it then becomes very important to recognize when a conjugacy class admits an exchange move.

In this paper we explore the question: how can we recognize whether the conjugacy class of a given closed braid admits a reducing move or an exchange move?

The question of detecting reducing moves was posed in [6]. In [23] McCool proved the existence of an algorithm for determining whether the conjugacy class of a braid admits a reducing move, using Garside's solution to the word 


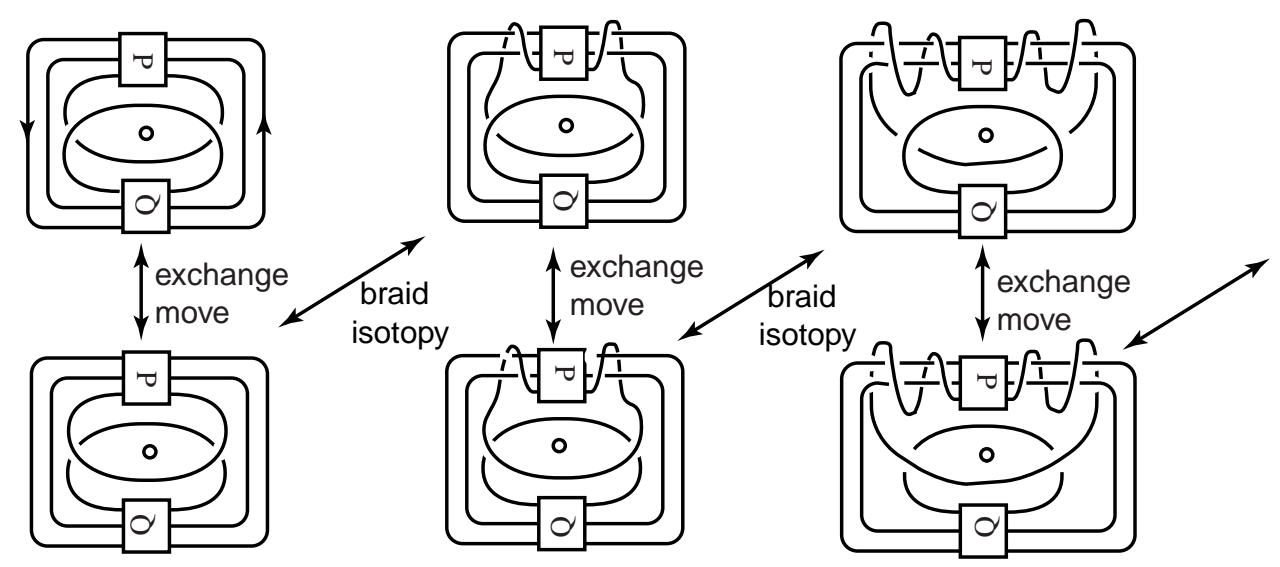

Figure 4: Exchange moves and braid isotopy can lead to infinitely many conjugacy classes of closed braid representatives of a knot or link

and conjugacy problems in $\mathbf{B}_{\mathbf{n}}$. The problems of recognizing reducing moves and exchange moves were considered by Fehrenbach in his (unpublished) $\mathrm{PhD}$ thesis [12, using an approach which is very different from ours. In 13] Fiedler gives a partial invariant which shows that the conjugacy class of a braid of braid index $\geq 4$ is in general changed by exchange moves. (For $n=3$ exchange moves can be realized by conjugacy, except in the special case of composite knots). To the best of our knowledge no algorithmic solution exists to the problem of recognizing when a braid conjugacy class admits an exchange move.

At the same time that the connections which we just described between braids and links were under investigation, a parallel but quite different set of investigations was revealing new information about matrix representations of the family of braid groups $\left\{\mathbf{B}_{n}, n \in \mathbb{Z}^{+}\right\}$. For many years a central problem about the braid groups had been the question of whether they were or were not linear groups. Inspired by the work of Thurston (see [11) on surface mapping class groups, it was shown in [8, 22] and also [15] that braid groups shared deep structural properties with linear groups. However, there was more to it than that. In the 1930's an especially interesting irreducible representation of $\mathbf{B}_{n}$ in $G L_{n-1}\left(\mathbb{Z}\left[t, t^{-1}\right]\right)$ had been introduced by W. Burau [10, and this Burau representation of $\mathbf{B}_{n}$ was widely regarded as a likely candidate for the sought-for faithful matrix representation. It was recognized by various people that the representation had a natural interpretation via the action of $\mathbf{B}_{n}$ on the $n$-times punctured plane $P_{n}$ which lifted to an action on $H_{1}\left(\tilde{P}_{n}, \mathbb{Z}\right)$ of the infinite cyclic covering space $\tilde{P}_{n}$ of $P_{n}$, where the covering translation gave a module structure to $H_{1}$. This fact enabled the second author of this paper to prove, in [25], that for sufficiently large $n$ the Burau representation is in fact not faithful. The key new fact which made that proof possible was the interpretation of the entries in the Burau matrix of a geometric braid $K$ as recording information about intersections between the lifts of certain $\operatorname{arcs}$ on $P_{n}$ and their images under a braid homeomorphism $\beta$ to $\tilde{P}_{n}$, and that the proof of faithfulness rested on whether one could construct two arcs which have essential geometric intersections, but tricked the Burau matrix into thinking they did not intersect. 
Non-faithfulness was then proved for $n \geq 9$ (resp. 6,5) in [25] (resp. 21], [1]).

At this juncture there may be a historic opportunity, as developments in both the geometry and the algebra are beginning to bridge the gap between the two techniques. Regarding the geometry, it is now possible to replace the Markov theory with the more powerful Birman-Menasco theory, a relevant portion of which was just described. As for the algebra, it is now known that there is no shortage of representations of $\mathbf{B}_{n}$ in subgroups of the linear group over a ring of Laurent polynomials (in general with more than 1 variable), moreover they sort themselves out into families in a way that suggests close connections with the Burau representation, but with one important difference: the newly discovered representations include ones that are faithful. We have in mind first the important work in Ruth Lawrence's thesis [19. Second, we are thinking of the key contribution from Daan Krammer, who rediscovered the Lawrence representation (in a different setting) and used it to prove the linearity of $\mathbf{B}_{4}$ in [17. Third, we have in mind the proof by S. Bigelow in [2] and D. Krammer in 18] that the 'Krammer Lawrence' or KL representation is faithful for all $n$. Finally, we have in mind the construction which was first suggested in 9] and then investigated in detail in 20]. As will be proved below, in the simplest case the latter construction gives a variation on the KL representation. Like the Burau representation, it detects intersections of certain arcs on the $n$-times punctures disc, and this is the fact that we exploit in relation to the problems in algorithmic knot and link theory that we discussed above.

The main result of this paper will be the development of algebraic techniques to detect when the conjugacy class of a closed braid which represents a knot admits a reducing or exchange move. See Theorems 2 and 3 and Corollary 1 Here is the plan of this paper. In Section 2 we establish our conventions and define the particular representations that we shall use here. In section 3 we define the intersection pairing and determine its properties. In Section 4 (resp. 5) we establish the relationship between the intersection pairing created by the action of a braid homeomorphism $\beta$ on the n-times punctured plane and the detection of reducing loops (resp. exchange moves) in the associated closed

braid. In Section [6] we establish the connection between the intersection pairing and certain blocks of zeros in the image of $\beta$ under our matrix representation of $\mathbf{B}_{n}$. In Section $\mathbf{7}$ we explain why our work does not, at this time, give an algorithm for recognizing the unknot. We discuss the open problems whose solution would make it into an algorithm. We also discuss several other interesting open problems which were suggested by the work in this paper.

\section{Representations of $\mathbf{B}_{n}$}

In this section we'll review the general construction of 'homology representations' of $\mathbf{B}_{n}$. We begin by establishing our conventions and notation. After that we construct the representation which is of primary interest, first in a special case, and then in a more general setting. Finally, we show why the special case gives a reducible form of the KL representation. 


\subsection{Braid homeomorphisms and geometric braids}

We will need to use two ways of looking at a closed braid: as a geometric closed braid $\tilde{K}$ in $\mathbb{R}^{3}$ (with a particular choice of a half-plane $P$ along which we cut it open to a braid $K$ ) and as a homeomorphisms $\beta$ of $P$, punctured at $n$ points.

We now explain our conventions for passing between $\beta$ and $K$. Let

$$
P=\left\{(x, y, z) \in \mathbb{R}^{3}: y=0, x \geq 0\right\}
$$

and let $A \subset P$ be the $z$-axis. Then there is a map $F: P \times[0,1] \rightarrow \mathbb{R}^{3}$ defined by

$$
F((x, 0, z), t)=(x \cos (2 \pi t), x \sin (2 \pi t), z) .
$$

Note that when $t=0$ or 1 the restriction of $F$ to $P$ is the identity map.

Choose a set of points $Q_{n}=\left\{q_{1}, \ldots, q_{n}\right\}$ on $P$ in the interior of a disc $D^{2} \subset P$. Let $\beta: P \rightarrow P$ be a braid homeomorphism, i.e. a homeomorphism of $P$ which fixes the set $Q_{n}$ and is the identity on $P \backslash \operatorname{int}(D)$. Then $\beta$ is isotopic to the identity as a homeomorphism of $P$ and so there is an isotopy (which we may assume is fixed on $P \backslash \operatorname{int}(D))$, say $H: P \times[0,1] \rightarrow P$, with

$H(p, 0)=p, H(p, 1)=\beta(p)$ for all $p \in P$. Then $H$ induces a homeomorphism $H^{\prime}: P \times[0,1] \rightarrow P \times[0,1]$ which is defined by $H^{\prime}(p, t)=(H(p, 1-t), 1-t)$. The composite map $\mathbf{H}=F \circ H^{\prime}$ induces identifications $\mathbf{H}(p, 0)=\mathbf{H}(p, t)$ if $p \in A$ and $\mathbf{H}(p, 1)=\mathbf{H}(\beta(p), 0)$ for all $p \in P$. Clearly $\mathbb{R}^{3}$, Euclidean space, is obtained from $P \times[0,1]$ by these identifications. The image of $Q_{n} \times[0,1]$ under $\mathbf{H}$ will be the closed geometric braid $\tilde{K}$ determined by the braid homeomorphism $\beta$. The fact that we have chosen the half-plane $P \times\{0\}=P \times\{1\}$ as our reference gives us, in a natural way, an open geometric braid associated to $\beta$ : it is obtained by cutting $\tilde{K}$ along the half plane $P \times\{0\}$. See the example in Figure 1

Now that we have learned how to pass between $\beta$ and $K$, we will not need to distinguish between them. Thus we may pass back and forth freely between the interpretation of our braid as a homeomorphism of the n-times punctured plane and as a geometric braid in 3 -space. We will use the symbol $\beta$ for elements of $\mathbf{B}_{n}$ and the symbol $\tilde{\beta}$ for the conjugacy class of $\beta$. This is the same as the isotopy class of the associated closed braid, where isotopy means isotopy in the complement in $\mathbb{R}^{3}$ of the braid axis.

\subsection{The group $\mathrm{B}_{1, n}$}

We will be interested in the braid group $\mathbf{B}_{n}$ on n-strands, but in order to look at $\mathbf{B}_{n}$ as a group of isotopy classes of homeomorphisms of the punctured plane it will be convenient to regard it as a subgroup of $\mathbf{B}_{n+1}$. Number the strands in the latter group as $0,1, \ldots, n$. Let $\mathbf{B}_{1, n} \subset \mathbf{B}_{n+1}$ be the subgroup of braids in $\mathbf{B}_{n+1}$ whose associated permutation fixes the letter 0 . Its relationship to $\mathbf{B}_{n}$ is given by the group extension

$$
1 \rightarrow \mathbf{F}_{n} \rightarrow \mathbf{B}_{1, n} \rightarrow \mathbf{B}_{n} \rightarrow 1,
$$

where the homomorphism $\mathbf{B}_{1, n} \rightarrow \mathbf{B}_{n}$ is defined by pulling out the zero ${ }^{\text {th }}$ braid strand. There is a cross section which is defined by mapping $\mathbf{B}_{n}$ to the subgroup of braids on strands $1, \ldots, n$ in $\mathbf{B}_{1, n}$. Therefore we may identify $\mathbf{B}_{1, n}$ with $\mathbf{F}_{n} \rtimes \mathbf{B}_{n}$. 
We use bold-faced roman letters for elements of $\mathbf{F}_{\mathbf{n}}$, Greek letters for elements of $\mathbf{B}_{\mathbf{n}}$, and upper case Roman letters for elements of $\mathbf{B}_{1, n}$. Since $\mathbf{F}_{n}$ and $\mathbf{B}_{n}$ are both known groups, and since the action of $\mathbf{B}_{n}$ of $\mathbf{F}_{n}$ is also known, we

conclude that $\mathbf{B}_{1, n}$ has a presentation with generators $\mathbf{x}_{\mathbf{1}}, \ldots, \mathbf{x}_{\mathbf{n}}, \sigma_{1}, \ldots, \sigma_{n-1}$, where the $\mathbf{x}_{\mathbf{i}}{ }^{\prime} s$ generate the free factor $\mathbf{F}_{n}$ and the elementary braids $\sigma_{j}$ generate the factor $\mathbf{B}_{n}$, satisfying the well-known braid relations:

$$
\sigma_{i} \sigma_{j}=\sigma_{j} \sigma_{i} \quad \text { if } \quad|i-j| \geq 2, \quad \text { and } \quad \sigma_{i} \sigma_{j} \sigma_{i}=\sigma_{j} \sigma_{i} \sigma_{j} \quad \text { if } \quad|i-j|=2 \text {. }
$$

If a word $\sigma_{\mu_{1}}^{\epsilon_{1}} \cdots \sigma_{\mu_{r}}^{\epsilon_{r}}$ represents an element of $\mathbf{B}_{\mathbf{n}}$, then $\sigma_{\mu_{1}}^{\epsilon_{1}}$ corresponds to the first crossing in the oriented braid.

Our conventions for $\mathbf{x}_{\mathbf{1}}, \ldots, \mathbf{x}_{\mathbf{n}}$ are that the generator $\mathbf{x}_{\mathbf{i}}$ represents a braid in which strand 0 begins at $q_{i} \times\{0\}$, travels in front of strands $1 \ldots, i-1$, then around strand $i$ with positive linking number, and then passes to $q_{i} \times\{1\}$ traveling in front of strands $1 \ldots, i-1$ again. The geometry then determines additional relations which give the action of $\mathbf{B}_{n}$ on $\mathbf{F}_{n}$ (3):

$$
\sigma_{i} \mathbf{x}_{\mathbf{j}} \sigma_{i}^{-1}= \begin{cases}\mathbf{x}_{\mathbf{i}+\mathbf{1}} & \text { if } j=i \\ \mathbf{x}_{\mathbf{i}+\mathbf{1}}{ }^{-1} \mathbf{x}_{\mathbf{i}} \mathbf{x}_{\mathbf{i}+\mathbf{1}} & \text { if } \mathbf{j}=\mathrm{i}+1 \\ \mathbf{x}_{\mathbf{j}} & \text { otherwise }\end{cases}
$$

In this way we see that the groups $\mathbf{B}_{n}$ and $\mathbf{F}_{n}$ are both subgroups of $\mathbf{B}_{1, n} \subset \mathbf{B}_{n+1}$.

\subsection{Magnus representations and an example}

In this section we show how the ideas which were introduced by W. Magnus to explain the underlying mechanism behind the Burau representation of $\mathbf{B}_{\mathbf{n}}$ (see 6] ) can be bootstrapped to yield new representations of $\mathbf{B}_{\mathbf{n}}$. Later we will see that our new, augmented representation is in fact the Lawrence-Krammer representation of $\mathbf{B}_{\mathbf{n}}$.

Recall that the free calculus was used by Gassner in [14] to construct an irreducible representation of the pure braid group $\mathbf{P}_{n+1}$ in the ring of $n$-dimensional matrices whose entries are Laurent polynomials in $n$ variables. In 6] it was explained how similar ideas could be used to construct representations of the subgroups of $\mathbf{B}_{n+1}$ which lie between $\mathbf{B}_{n+1}$ and $\mathbf{P}_{n+1}$. The one that interests us here is the irreducible representation of the in-between subgroup $\mathbf{B}_{1, n}=\mathbf{F}_{n} \rtimes \mathbf{B}_{n}$ over the ring $\mathbb{Z}\left[t, t^{-1}, q, q^{-1}\right]$ of Laurent polynomials in 2 variables. The variable $t$ is to be thought of as being associated with strand 0 and the variable $q$ as being associated to strands 1 through $n$. Call this the Magnus representation of $\mathbf{B}_{1, n}$. Let $\mathcal{M}$ be the ring of $n+1$ by $n+1$ matrices with entries in $\mathbb{Z}\left[t, t^{-1}, q, q^{-1}\right]$. The Magnus representation is a representation

$$
\rho: \mathbf{F}_{n} \rtimes \mathbf{B}_{n} \rightarrow \mathcal{M}
$$

It has dimension $(n+1) \times(n+1)$, but as is well known it's is reducible to $n \times n$. For our purposes it will be most convenient to use the unreduced form, and we shall do so. 
It is shown in [6] how to construct the images of the generators of $\mathbf{F}_{n} \rtimes \mathbf{B}_{n}$ under $\rho$. Working out an example, we obtain the following matrices for the generators of $\rho\left(\mathbf{F}_{n} \rtimes \mathbf{B}_{n}\right)$ in the case $n=4$ :

$$
\begin{gathered}
\sigma_{1} \mapsto\left(\begin{array}{ccccc}
1 & 0 & 0 & 0 & 0 \\
0 & 0 & q & 0 & 0 \\
0 & 1 & 1-q & 0 & 0 \\
0 & 0 & 0 & 1 & 0 \\
0 & 0 & 0 & 0 & 1
\end{array}\right), \quad \sigma_{2} \mapsto\left(\begin{array}{ccccc}
1 & 0 & 0 & 0 & 0 \\
0 & 1 & 0 & 0 & 0 \\
0 & 0 & 0 & q & 0 \\
0 & 0 & 1 & 1-q & 0 \\
0 & 0 & 0 & 0 & 1
\end{array}\right) \\
\sigma_{3} \mapsto\left(\begin{array}{ccccc}
1 & 0 & 0 & 0 & 0 \\
0 & 1 & 0 & 0 & 0 \\
0 & 0 & 1 & 0 & 0 \\
0 & 0 & 0 & 0 & q \\
0 & 0 & 0 & 1 & 1-q
\end{array}\right)
\end{gathered}
$$

and

$$
\begin{aligned}
& x_{1} \mapsto\left(\begin{array}{ccccc}
q & -q(1-q) & 0 & 0 & 0 \\
1-t & 1+t q-q & 0 & 0 & 0 \\
0 & 0 & 1 & 0 & 0 \\
0 & 0 & 0 & 1 & 0 \\
0 & 0 & 0 & 0 & 1
\end{array}\right) \\
& x_{2} \mapsto\left(\begin{array}{ccccc}
q & -(1-q)^{2} & -q(1-q) & 0 & 0 \\
0 & 1 & 0 & 0 & 0 \\
1-t & (1-t)(1-q) & 1-q+t q & 0 & 0 \\
0 & 0 & 0 & 1 & 0 \\
0 & 0 & 0 & 0 & 1
\end{array}\right), \\
& x_{3} \mapsto\left(\begin{array}{ccccc}
q & -(1-q)^{2} & -(1-q)^{2} & -q(1-q) & 0 \\
0 & 1 & 0 & 0 & 0 \\
0 & 0 & 1 & 0 & 0 \\
1-t & (1-t)(1-q) & (1-t)(1-q) & 1-q+t q & 0 \\
0 & 0 & 0 & 0 & 1
\end{array}\right), \\
& x_{4} \rightarrow\left(\begin{array}{ccccc}
q & -(1-q)^{2} & -(1-q)^{2} & -(1-q)^{2} & -q(1-q) \\
0 & 1 & 0 & 0 & 0 \\
0 & 0 & 1 & 0 & 0 \\
0 & 0 & 0 & 1 & 0 \\
1-t & (1-t)(1-q) & (1-t)(1-q) & (1-t)(1-q) & 1-q+t q
\end{array}\right) .
\end{aligned}
$$

Let $\mathcal{D}(z)$ denote a diagonal matrix whose diagonal entrises are $z$. We next define the homomorphism

$$
\tau: \mathbf{F}_{n} \rtimes \mathbf{B}_{n} \rightarrow \mathcal{M} \quad \text { by } \quad \tau(G)=\mathcal{D}\left(q^{\operatorname{deg}(G)}\right) \rho(G),
$$

where $G \in \mathbf{B}_{1, n}$ and $\operatorname{deg}(G)$ denotes the degree of $G$, i.e. its exponent sum in the basis elements $\mathbf{x}_{\mathbf{i}}$ of the free group $\mathbf{F}_{\mathbf{n}}$. In particular, $\tau\left(\sigma_{i}\right)=\rho\left(\sigma_{i}\right)$, whereas $\tau\left(\mathbf{x}_{\mathbf{j}}\right)=\mathcal{D}(q) \rho\left(\mathbf{x}_{\mathbf{j}}\right)$.

Using the representation $\tau: \mathbf{B}_{1, n} \rightarrow \mathcal{M}$, we are finally ready to construct (in our special case) the representation $\tau^{+}$of $\mathbf{B}_{n}$ which is of primary interest to us 
in this paper. It is essentially identical to Lawrence's version (see [18]) of the now-famous Krammer-Lawrence representation. Let $P_{n}=P-Q_{n}$ denote the $n$-times punctured plane. View $F_{n}$ as the fundamental group of $P_{n}$ based at some point $x \in A=\partial P_{n}$, and use the representation $\tau$ (restricted to the first factor) to define a local system $\mathcal{L}$ on $P_{n}$. We already gave the action of $\mathbf{B}_{\mathbf{n}}$ on $\mathbf{F}_{\mathbf{n}}$ in (3). It will be helpful to think of a local system as a covering space which has the structure of a fiber bundle, where the fibers are copies of the Magnus reprsentation $V=\mathbb{Z}\left[t, t^{-1}, q, q^{-1}\right]^{n}$. The homology $H_{1}\left(P_{n}, x, \mathcal{L}\right)$ is sometimes called $H_{1}\left(P_{n}, x, V\right)$, homology with coefficients in $V$ viewed as a module over the fundamental group of $P_{n}$. It is a direct sum $V \oplus V \oplus \ldots \oplus V$ ( $n$ factors) and we denote by $e_{i}: V \rightarrow H_{1}\left(P_{n}, x, \mathcal{L}\right)$ the inclusion on the $i$ th factor. For $\alpha \in V$ the identification is such that $e_{i}(\alpha)$ is the loop $x_{i}$ lifted to the cover, in such a way that the basepoint $x$ at the beginning lifts to the element $\alpha \in V$.

We use the action (3) to compute the map induced by the elementary braid generators $\sigma_{i}$ on homology:

$$
\sigma_{i}: H_{1}\left(P_{n}, x, \mathcal{L}\right) \rightarrow H_{1}\left(P_{n}, x,\left(\sigma_{i}\right)_{*}(\mathcal{L})\right) .
$$

Since it is enough to determine the homology classes $\sigma_{i}\left(e_{j}\right)(\alpha)$ for $\alpha \in V$ it is enough to calcluate the composites $\sigma_{i} e_{j}$. Identifying both source and target of the map with $V \oplus V \oplus \ldots \oplus V$ in the manner described above, we find:

$$
\sigma_{i} e_{j}= \begin{cases}e_{j+1}\left(\tau\left(\mathbf{x}_{j+1}\right)\right) & \text { if } j=i \\ e_{i}\left(\tau\left(\mathbf{x}_{i}\right)\right)+e_{i+1}\left(1-\tau\left(\mathbf{x}_{i+1}\right)\right) & \text { if } j=i+1 \\ e_{j} & \text { otherwise }\end{cases}
$$

To understand (5), we think of $e_{i+1}(\alpha)$ as the loop $x_{i+1}$ lifted to the cover, in such a way that the base point $x$ at the beginning lifts to the element $\alpha \in V$. When we apply $\sigma_{i}$ we get a loop that traces $x_{i+1}$ anticlockwise, then $x_{i}$ anticlockwise, and finally $x_{i+1}$ clockwise. However, the initial points of these loops lift to three different sheets in the cover, i.e. to three different elements of elements of $V$. These are the coefficients of $e_{i+1}, e_{i}$, and $-e_{i+1}$, taking account of the fact that the action of a loop $w$ in $P_{n}$ on $\left(\sigma_{i}\right)_{*}(\mathcal{L})$ is the action of $\sigma_{i}(w)$ on $\mathcal{L}$.

Note that in future sections we will make the following identification. Since $\mathcal{M}=\operatorname{End}(V)$ is the endomorphism ring of $V$, The map $e_{i}: V \rightarrow H_{1}\left(P_{n}, x, V\right)$ can be viewed as an element of $H_{1}\left(P_{n}, x, \mathcal{M}\right)$ where the homology is taken with respect to the left action of $F_{n}$ on $\mathcal{M}$. This homology module is a free right $\mathcal{M}$ module of rank $n$ and the $e_{i}$ are a basis for this module. The formula displayed above describes the action of $\sigma_{i}$ with respect to this basis. However we are not quite done. The resulting matrix with $\mathcal{M}$ entries does not define a representation because our identification of the source and target both with $V \oplus V \oplus \ldots \oplus V$ was not natural.

For any braid $\beta$ there is a natural map $\beta_{*} \mathcal{L} \rightarrow \mathcal{L}$ which is given on the fiber $V$ over $x$ by multiplication by $\tau(\beta)$, and it extends to a map of the whole local system because of the relations (3) in $\mathbf{B}_{1, n}$. Now let $\alpha$ be any other braid. Applying $\alpha_{*}$ we obtain a second map of local systems $\alpha_{*} \beta_{*} \mathcal{L} \rightarrow \alpha_{*} \mathcal{L}$ Because 
homology is a bifunctor we then obtain a commutative square

$$
\begin{array}{cccc}
H_{1}\left(P_{n}, x, \mathcal{L}\right) \rightarrow & H_{1}\left(P_{n}, x, \beta_{*} \mathcal{L}\right) & \rightarrow & H_{1}\left(P_{n}, x, \alpha_{*} \beta_{*} \mathcal{L}\right) \\
& & \downarrow \\
& & \\
& H_{1}\left(P_{n}, x, \mathcal{L}\right) & \rightarrow & H_{1}\left(P_{n}, x, \alpha_{*} \mathcal{L}\right) . \\
& & \downarrow \\
& & & H_{1}\left(P_{n}, x, \mathcal{L}\right)
\end{array}
$$

Each module in the diagram is a direct sum of $n$ copies of $V$ and so each map may be represented by an $n$ by $n$ matrix with entries in $\mathcal{M}$. The leftmost vertical map in the square is given by a diagonal matrix $\mathcal{D}(\tau(\beta))$ with each entry equal to $\tau(\beta)$. The horizontal maps are the maps on homology given on generators by formula (5). The fact that the square commutes implies an equality of two maps from the upper left corner to the lower right corner $\mathcal{D}(\tau(\alpha)) \alpha \mathcal{D}(\tau(\beta)) \beta=\mathcal{D}(\tau(\alpha \beta)) \alpha \beta$, so the assignment of each braid generator $\sigma_{i}$ to the matrix $\mathcal{D}\left(\sigma_{i}\right) \sigma_{i}$ describes a braid group representation. Thus, if $\mathcal{D}\left(\tau\left(\sigma_{i}\right)\right), i=1,2,3$ denotes the block diagonal matrix (with each block a $5 \times 5$ matrix) whose diagonal entries are the blocks $\tau\left(\sigma_{i}\right)$, defined earlier, the 'augmented' representation $\tau^{+}$of $\mathbf{B}_{4}$ is given on the elementary braid generators by:

$$
\begin{aligned}
& \tau^{+}\left(\sigma_{1}\right)=\mathcal{D}\left(\tau\left(\sigma_{1}\right)\right)\left(\begin{array}{cccc}
0 & \tau\left(x_{1}\right) & 0 & 0 \\
1 & 1-\tau\left(x_{2}\right) & 0 & 0 \\
0 & 0 & 1 & 0 \\
0 & 0 & 0 & 1
\end{array}\right)= \\
& \left(\begin{array}{cccc}
0 & \tau\left(\sigma_{1}\right) \tau\left(x_{1}\right) & 0 & 0 \\
\tau\left(\sigma_{1}\right) & \tau\left(\sigma_{1}\right)\left(1-\tau\left(x_{2}\right)\right) & 0 & 0 \\
0 & 0 & \tau\left(\sigma_{1}\right) & 0 \\
0 & 0 & 0 & \tau\left(\sigma_{1}\right)
\end{array}\right) \\
& \tau^{+}\left(\sigma_{2}\right)=\mathcal{D}\left(\tau\left(\sigma_{2}\right)\right)\left(\begin{array}{cccc}
1 & 0 & 0 & 0 \\
0 & 0 & \tau\left(x_{2}\right) & 0 \\
0 & 1 & 1-\tau\left(x_{3}\right) & 0 \\
0 & 0 & 0 & 1
\end{array}\right)= \\
& \left(\begin{array}{cccc}
\tau\left(\sigma_{2}\right) & 0 & 0 & 0 \\
0 & 0 & \tau\left(\sigma_{2}\right) \tau\left(x_{2}\right) & 0 \\
0 & \tau\left(\sigma_{2}\right) & \tau\left(\sigma_{2}\right)\left(1-\tau\left(x_{3}\right)\right) & 0 \\
0 & 0 & 0 & \tau\left(\sigma_{2}\right)
\end{array}\right) \\
& \tau^{+}\left(\sigma_{3}\right)=\mathcal{D}\left(\tau\left(\sigma_{3}\right)\right)\left(\begin{array}{cccc}
1 & 0 & 0 & 0 \\
0 & 1 & 0 & 0 \\
0 & 0 & 0 & \tau\left(x_{3}\right) \\
0 & 0 & 1 & 1-\tau\left(x_{4}\right)
\end{array}\right)= \\
& \left(\begin{array}{cccc}
\tau\left(\sigma_{3}\right) & 0 & 0 & 0 \\
0 & \tau\left(\sigma_{3}\right) & 0 & 0 \\
0 & 0 & 0 & \tau\left(\sigma_{3}\right) \tau\left(x_{3}\right) \\
0 & 0 & \tau\left(\sigma_{3}\right) & \tau\left(\sigma_{3}\right)\left(1-\tau\left(x_{4}\right)\right)
\end{array}\right)
\end{aligned}
$$

The rank of this representation is $4 \times 5=20$ (for general $n$ it is $n(n+1)$ ). If we had used the reduced Magnus matrices the rank would have been $n^{2}$. 


\subsection{The general case}

Suppose we are given a representation $\tau: \mathbf{B}_{1, n} \rightarrow G L(V)$. The module $H_{1}\left(P_{n}, x, \mathcal{L}\right)$ has a $\mathbf{B}_{n}$ action, coming from the fibration

$$
P_{n} \rightarrow X_{1, n} \rightarrow X_{n}
$$

where $X_{1, n}$ is the space of $n$ distinct points in $P$ with one marked point, and $X_{n}$ is the space of $n$ distinct points in $P$. The second map is forgetting the marked point. The fiber over a subset $Q_{n} \subset P$ is $P_{n}$. The action of $\mathbf{B}_{1, n}=\pi_{1}\left(X_{1, n}\right)$ on $V$ is taken as the monodromy defining a local system on $X_{1, n}$ and as usual the fundamental group of the base acts on the homology of the fiber over $Q_{n}$. Note that the group $H_{1}\left(P_{n}, x, \mathcal{L}\right)$ is homology relative to the basepoint. This is a direct sum of $n$ copies of $V$. The action of a braid $\beta$ is the composite of the homology action $H_{1}\left(P_{n}, x, \mathcal{L}\right) \rightarrow H_{1}\left(P_{n}, x, \beta_{*} \mathcal{L}\right)$ with the map induced on homology by $\beta_{*} \mathcal{L} \rightarrow \mathcal{L}$, which multiplies the fiber $V$ over $x$ by $\tau(\beta)$. Thus, given a representation $\tau: \mathbf{B}_{1, n} \rightarrow G L(V)$ one obtains immediately a new representation $\tau^{+}: \mathbf{B}_{n} \rightarrow G L(V \oplus \ldots \oplus V)$.

\subsection{The Krammer-Lawrence representation}

We now show that our $n(n+1)$ dimensional representation contains the one Krammer and Bigelow have proven faithful (with our ' $t$ ' playing the role of Krammer's $t^{2}$ ). Although we've displayed the unreduced Magnus matrices above, for the moment let us take $\rho: \mathbf{B}_{1, n} \rightarrow \mathcal{M}$ to be the size $n$ reduced matrices. Let $X_{n}$ be the set of $n$ element subsets $Q_{n} \subset P$, let $X_{1, n}$ be the set of subsets $\left\{q_{0}, \ldots, q_{n}\right\} \subset P$ with $q_{0}$ marked, and let $X_{2, n}$ be the set of subsets of order $n+2$ of $P$ with two separately marked points. Recall $Q_{n}=\left\{q_{1}, \ldots, q_{n}\right\}$ and $P_{n}=P-Q_{n}$. Let $S P$ be the space of pairs of distinct, separately marked points in $P_{n}$. Let $\mathcal{L}$ be the local system of $X_{2, n}$ with fiber $\mathbb{Z}\left[t, t^{-1}, q, q^{-1}\right]$ and monodromy matrices as in Krammer's and Bigelow's papers, so that the power of $q$ is the winding number of either marked point around $q_{1}, \ldots, q_{n}$ and the power of $t$ is the winding number between the marked points. We have the diagram of fibrations

$$
\begin{array}{ccccc}
\left(P-\left\{q_{0}, . ., q_{n}\right\}\right) & \rightarrow & S P & \rightarrow & P-\left\{q_{1}, . ., q_{n}\right\} \\
\downarrow & & i \downarrow & & k \downarrow \\
\left(P-\left\{q_{0}, \ldots, q_{n}\right\}\right) & \stackrel{j}{\rightarrow} & X_{2, n} & \rightarrow & X_{1, n} \\
& & \downarrow & & \downarrow \\
& & X_{n} & \rightarrow & X_{n}
\end{array}
$$

The representation of $B_{n}$ defined by $\tau^{+}$maps onto $V$ and the kernel is a copy of the unreduced homology $H_{1}\left(P_{n}, k^{*} \mathcal{H}\right)$ where $\mathcal{H}$ is the local system on $X_{1, n}$ corresponding to the representation $\tau: \mathbf{B}_{1, n} \rightarrow \mathcal{M}$. We wish to compare this with the Krammer Lawrence representation of $B_{n}$. From the spectral sequence of a fibration we have an isomorphism of representations of the fundamental group $B_{n}$ of $X_{n}$.

$$
H_{2}\left(S P, i^{*} \mathcal{L}\right)=H_{1}\left(P_{n}, k^{*} \mathcal{H}_{1}\left(P-\left\{q_{0}, \ldots, q_{n}\right\}, j^{*} \mathcal{L}\right)\right)
$$

The module on the left is the Krammer-Lawrence representation (but with two marked points instead of two indistinguishable points) while module on the right is the homology $H_{1}\left(P_{n}, k^{*} \mathcal{H}^{\prime}\right)$ with coefficients in the local system 
$\mathcal{H}^{\prime}=\mathcal{H}_{1}\left(P-\left\{q_{0}, \ldots, q_{n}\right\}, j^{*} \mathcal{L}\right)$. To prove $H_{1}\left(P_{n}, k^{*} \mathcal{H}\right)$ is isomorphic to $H_{1}\left(P_{n}, k^{*} \mathcal{H}^{\prime}\right)$ it now remains only to identify the two local systems $\mathcal{H}$ and $\mathcal{H}^{\prime}$ on $X_{1, n}$. For $G \in \mathbf{B}_{1, n}$ the monodromy of $G$ on the first local system is $\tau(G)$. The monodromy on the second is the composite of the homology map $H_{1}\left(P-\left\{q_{0}, \ldots, q_{n}\right\}, j^{*} \mathcal{L}\right) \rightarrow H_{1}\left(P-\left\{q_{0}, \ldots, q_{n}\right\}, g_{*} j^{*} \mathcal{L}\right)$, represented by the reduced Magnus matrix $\rho(G)$, followed by the map on homology induced by $\beta_{*} j^{*} \mathcal{L} \rightarrow j^{*} \mathcal{L}$, which is multiplication by $q^{\operatorname{deg}(G)}$. The composite is $\tau(G)$, as needed. To get the Krammer-Lawrence representation of two unmarked points it is necessary to adjoin $\sqrt{t}$ to our coefficient ring (remember our $t$ is Krammer's $t^{2}$ ). The local system $\mathcal{L}$ is now the pullback of a local system on $X_{2, n} / C_{2}$ where the cyclic group $C_{2}$ acts freely by interchanging the markings on the two points. We obtain a $C_{2}$ action on $H_{2}(S P)$ and since the Krammer-Lawrence representation has no 2-torsion so we may identify it with the $C_{2}$ invariant submodule, which is therefore faithful, from which $\tau^{+}$is as well.

Note too that the $\tau^{+}$are all faithful (because the Lawrence-Krammer representation has been proved to be faithful). On the other hand, the $\tau$ are not all faithful (because the $\tau\left(\sigma_{i}\right)$ act on the last $n$ basis vectors exactly as the Burau matrices do. ) These two facts will be important in Theorem 1 below.

\section{The intersection pairing}

For the rest of this section, $\mathcal{M}$ will denote an arbitrary ring, depending on $n$, together with a homomorphism

$$
\tau: \mathbf{B}_{1, n} \rightarrow \mathcal{M}
$$

to the units of $\mathcal{M}$. We further assume that the $\operatorname{rings} \mathcal{M}$ are appropriately nested as $n$ increases.

In the theorem which is stated below we will use the representation $\tau: \mathbf{B}_{1, n} \rightarrow \mathcal{M}$ to allow us to multiply elements of $\mathcal{M}$ on the left or on the right by elements of $\mathbf{B}_{n}$ or $\mathbf{F}_{n}$. In order to define fundamental classes of $\operatorname{arcs}$ in $P_{n}$ we work with homology with coefficients in $\mathcal{M}$. Choose points $x, y$ on the oriented braid axis $A$. In the orientation of $A$ as the boundary of $P$ we choose $y$ next to $x$ (as in Figure 5), but with $y$ displaced in the positive direction compared to $x$ using the orientation on $\mathbf{A}$. Note that the axis $\mathbf{A}$ appears to go from right to left (the clockwise direction) in the figure. This means if we use the orientation on $\mathbf{A}$ to orient $P_{n}$ the figure shows the negative side of the plane $P_{n}$. This corresponds also to the negative side of the plane $P_{n} \times\{0\}$ in figure

I(d), such that the $\mathbf{A}$-axis in figure 5 corresponds to the upward-pointing $z$-axis in figure 1(d). In that figure the basepoints $x$ and $y$ would appear with $y$ above $x$ on the $z$-axis.

Because the fundamental group of $P_{n}$ maps to the units of the ring $\mathcal{M}$ it acts on any right or left $\mathcal{M}$ module. We denote by $\mathcal{M}_{\text {left }}$ the free rank one $\mathcal{M}$ module upon which $\mathcal{M}$ acts on the left by right $\mathcal{M}$ module homomorphisms, and we denote by $\mathcal{M}_{\text {right }}$ the free rank one module upon which $\mathcal{M}$ acts on the right by left $\mathcal{M}$ module homomorphisms. Thus we consider two separate homology modules:

$$
H_{1}\left(P_{n}, y, \mathcal{M}_{\text {right }}\right) \quad \text { and } \quad H_{1}\left(P_{n}, x, \mathcal{M}_{\text {left }}\right)
$$


The punctured plane is homotopy-equivalent to a wedge of loops based at $x$ or $y$ which encircle and separate the $n$ points, and since we are taking homology relative to a point, the module $H_{1}\left(P_{n}, x, \mathcal{M}_{\text {left }}\right)$ is equal to the cellular 1-chains of this wedge of circles. This is a right $\mathcal{M}$ module which is free of rank $n$. It is a two-sided $\mathbf{B}_{n}$ module, with the right action coming from the fact that $\mathbf{B}_{n}$ maps to $\mathcal{M}$ and $\mathcal{M}$ acts on the right, the left action being the 'interesting' homology action, which commutes with the right $\mathcal{M}$ action. We can also form $H_{1}\left(P_{n}, y, \mathcal{M}_{\text {right }}\right)$ in the analogous way. It is a two-sided $\mathbf{B}_{n}$ action with the right action being the 'interesting' one. (Look ahead to formulas (12) and (13), also (14) and (15), where the actions are worked out explicitly). If $\gamma$ is a loop in $P_{n}$ based at $x$ we have a fundamental homology class $[\gamma]_{x} \in H_{1}\left(P_{n}, x, \mathcal{M}_{\text {left }}\right)$ and if $\delta$ is based at $y$ we have $[\delta]_{y} \in H_{1}\left(P_{n}, y, \mathcal{M}_{\text {right }}\right)$.

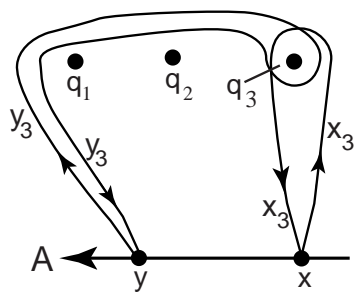

(a)

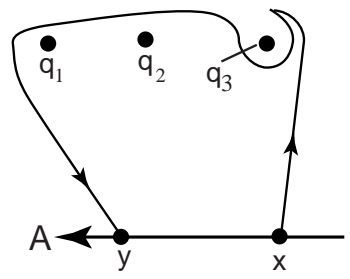

(b)

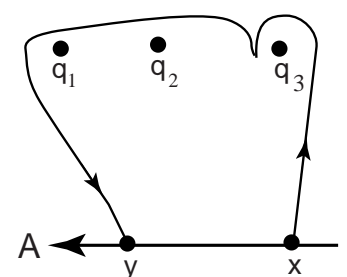

(c)

Figure 5: The intersection form

Note that our choice of notation implies that for $v \in H_{1}\left(P_{n}, x, \mathcal{M}_{\text {left }}\right)$ and braids $\alpha, \beta \in \mathbf{B}_{\mathbf{n}}$ the symbol

$$
\alpha v \beta
$$

means the same thing as $\tau^{+}(\alpha) v \tau(\beta)$ because the $\tau^{+}$representation defines the left action while the $\tau$ representation defines the right action. Also if $\mathbf{x} \in \mathbf{F}_{\mathbf{n}}$ the symbol

$v \mathbf{x}$

will always mean $v \tau(\mathbf{x})$. On the other hand if $w \in H_{1}\left(P_{n}, y, \mathcal{M}_{\text {right }}\right)$ then in the symbol

$$
\alpha w \beta
$$

it is the left action that is defined via $\tau(\alpha)$ and the right action is one which we have not named. It will turn out to be the transform of $\tau^{+}\left(\beta^{-1}\right)$ via a certain correspondence $\star$ but let us not worry about that yet. And if $\mathbf{x} \in \mathbf{F}_{\mathbf{n}}$ the symbol

$$
\mathbf{x} w
$$

will always mean $\tau(\mathbf{x}) w$.

As before, the free group $\mathbf{F}_{\mathbf{n}}$ will be viewed as the fundamental group of $P_{n}$. An element $\mathbf{w} \in \mathbf{F}_{\mathbf{n}}$ can be represented by a loop $w$ based at either point, as we shall identify the fundamental group of $P_{n}$ based at either $x$ or $y$ via a path in the axis $A$ connecting $x$ with $y$. If we choose $w$ to be a loop based at $x$ or at $y$ then we obtain two separate homology classes $[w]_{x}$ and $[w]_{y}$. Note that these belong to totally different homology groups. We may omit the subscript when there is no ambiguity. The elements $\mathbf{x}_{\mathbf{1}}, \ldots, \mathbf{x}_{\mathbf{n}}$ will be a basis of $F_{n}$ represented 
by $\operatorname{arcs} x_{1}, \ldots, x_{n}$ which begin and end at $x$, encircling the $q_{i}$ in such a way that the product $\mathbf{x}_{\mathbf{1}} \ldots \mathbf{x}_{\mathbf{n}}$ is represented by a simple arc $x_{1} \ldots x_{n}$ which encircles all the $q_{i}$. It is worthwhile to be quite careful about the orientations of the $x_{i}$. In all our figures, in which we display the negative side of $P_{n}$ with the axis $A$ at the bottom of the page, going from right to left, the $x_{i}$ are oriented so they appear to encircle $q_{i}$ in the counter-clockwise direction. We use the 'composition convention' for composing paths so that in the path $x_{1} x_{2}$ the point traverses first $x_{2}$ and later $x_{1}$.

It is convenient to introduce a second basis $\mathbf{y}_{\mathbf{1}}, \ldots, \mathbf{y}_{\mathbf{n}}$ of $F_{n}$ where $\mathbf{y}_{\mathbf{i}}=\mathbf{x}_{1} \mathbf{x}_{\mathbf{2}} \ldots \mathbf{x}_{\mathbf{i}-\mathbf{1}} \mathbf{x}_{\mathbf{i}}^{-1} \mathbf{x}_{\mathbf{i}-\mathbf{1}}^{-1} \ldots \mathbf{x}_{\mathbf{1}}^{-1}$. We shall usually represent the $\mathbf{y}_{\mathbf{i}}$ by arcs $y_{i}$ based at $y$. Note there is an involution of $\mathbf{F}_{\mathbf{n}}$ which interchanges the roles of the $\mathbf{x}_{\mathbf{i}}$ and the $\mathbf{y}_{\mathbf{i}}$.

In the statement below, a 'loop based at $x$ (resp $y$ )' will mean any loop encircling just a single $q_{i}$ which is oriented anticlockwise (resp. clockwise) when viewed on the negative side of the plane $P$. A 'simple homology class' will mean the class of a loop based at $x$ (respectively $y$ ) which has the additional property that it has no self-intersections.

Assume that we have been given a nested series of rings $\mathcal{M}$ (subscripts omitted) and representations $\tau: \mathbf{B}_{1, n} \rightarrow \mathcal{M}$ which satisfy the hypotheses:

- All $\tau^{+}: \mathbf{B}_{n} \rightarrow G L_{n}(\mathcal{M})$ are faithful,

- The representations $\tau=\tau_{n}: B_{1, n} \rightarrow \mathcal{M}$ are not all faithful, i.e. for some $n$ there is a non-trivial element in the kernel of $\tau_{n}$,

- There is an anti-involution $*$ on each $\mathcal{M}$ such that $\tau(G)^{*}=\tau\left(G^{-1}\right)$ for $G \in \mathbf{B}_{1, n}$,

- The $\tau\left(\mathbf{x}_{\mathbf{i}}\right)-1$ are non-zero-divisors in $\mathcal{M}$ which do not generate the unit ideal in the subring of $\mathcal{M}$ generated by the $\tau\left(\mathbf{x}_{\mathbf{i}}\right)$ and $\tau\left(\mathbf{x}_{\mathbf{i}}\right)^{-1}$.

Theorem 1 Under the hypotheses just stated, there is a generically non-degenerate $\mathcal{M}$ bilinear intersection pairing

$$
\langle,\rangle: H_{1}\left(P_{n}, y, \mathcal{M}_{\text {right }}\right\rangle \times H_{1}\left(P_{n}, x, \mathcal{M}_{\text {left }}\right) \rightarrow \mathcal{M}
$$

which has the following properties:

(a) If $\beta \in \mathbf{B}_{n}, v \in H_{1}\left(P_{n}, x, \mathcal{M}_{\text {left }}\right)$ and $w \in H_{1}\left(P_{n}, y, \mathcal{M}_{\text {right }}\right)$ then $\left\langle\beta v \beta^{-1}, \beta w \beta^{-1}\right\rangle=\beta\langle v, w\rangle \beta^{-1}$.

If in addition $u, s \in \mathcal{M}$ then $\langle u v, w s\rangle=u\langle v, w\rangle s$.

(b) If $\beta \in \mathbf{B}_{n}, v \in H_{1}\left(P_{n}, x, \mathcal{M}_{\text {left }}\right)$ and $w \in H_{1}\left(P_{n}, y, \mathcal{M}_{\text {right }}\right)$ then $\langle v \beta, w\rangle=\langle v, \beta w\rangle$.

(c) If $\gamma, \delta$ are loops based at $x, y$ respectively and $\beta \in \mathbf{B}_{n}$, then:

$$
\begin{gathered}
{[\beta \gamma]_{x}=\beta[\gamma]_{x} \beta^{-1} \in H_{1}\left(P_{n}, x, \mathcal{M}_{\text {left }}\right) \quad \text { and }} \\
{[\beta \delta]_{y}=\beta[\delta]_{y} \beta^{-1} \in H_{1}\left(P_{n}, y, \mathcal{M}_{\text {right }}\right) .}
\end{gathered}
$$


(d) There is a semilinear correspondence $\star$ between $H_{1}\left(P_{n}, y, \mathcal{M}_{\text {right }}\right)$ and $H_{1}\left(P_{n}, x, \mathcal{M}_{\text {left }}\right)$. It takes simple homology elements to simple homology elements and is compatible with the anti-involution $*$ on $\mathcal{M}$ and with the $B_{n}$ action, so that for $r \in \mathcal{M}, v \in H_{1}\left(P_{n}, x, \mathcal{M}_{\text {left }}\right) w \in H_{1}\left(P_{n}, y, \mathcal{M}_{\text {right }}\right)$ and $\beta \in B_{n}$ we have

$$
\begin{gathered}
(\beta v r)^{\star}=r^{*} v^{\star} \beta^{-1} \text { and } \\
(r w \beta)^{\star}=\beta^{-1} w^{\star} r^{*}
\end{gathered}
$$

Also for $\mathbf{w} \in \mathbf{F}_{n}$ if $w_{x}$ and $w_{y}$ are loops based at $x$ and $y$ respectively which represent $w$ then $\left[w_{x}\right]_{x}^{\star}=\left[w_{y}^{-1}\right]_{y}, \quad$ and $\quad\left[w_{y}\right]_{y}^{\star}=\left[w_{x}^{-1}\right]_{x}$.

(e) Let $\mathbf{w} \in \mathbf{F}_{n}$. Let $w$ be a loop based at $x$ which represents $\mathbf{w}$. Let $v=[w]_{x}$. Then a necessary condition for the existence of a simple arc representing $\mathbf{w}$ is:

$$
\left\langle v^{*}, v\right\rangle=\tau(\mathbf{w})-1 .
$$

where 1 denotes the identity in $\mathcal{M}$.

(f) If $\gamma, \delta$ are simple loops based at $x$ and $y$ respectively then $\left\langle[\delta]_{y},[\gamma]_{x}\right\rangle=0$ if and only if $\gamma$ and $\delta$ can be homotoped, keeping their endpoints fixed, so that they do not intersect. That is, the pairing is effective.

Proof: We begin our proof by constructing the intersection pairing, first via an example which will allow us to understand both the geometry and the algebra, and then algebraically, in full generality.

Construction of the pairing: We have not yet constructed the pairing, but it may be helpful to the reader to have an example to keep in mind before we do so. Consult Figure 5 (a). We wish to compute $\left\langle\left[y_{3}\right]_{y},\left[x_{3}\right]_{x}\right\rangle$. There are two points of intersection between $x_{3}$ and $y_{3}$. Travel along $x_{3}$, with its given orientation, to either intersection point and then switch to $y_{3}$ to avoid the intersection, preserving orientation. This determines an oriented arc from $x$ to $y$ for each intersection point. The arcs in the example are shown in sketches (b) and (c). They are given by the elements $\mathbf{x}_{1} \mathbf{x}_{2} \mathbf{x}_{3}$ and $\mathbf{x}_{1} \mathbf{x}_{\mathbf{2}}$ of $\mathbf{F}_{\mathbf{n}}$, where we read words from right to left (in other words we compose paths according to the conventions of function composition). The intersection is counted as positive (resp. negative) if it is necessary to make a right (resp. left) turn at the intersection point to avoid the intersection and preserve orientation. In the example $\left\langle\left[y_{3}\right]_{y},\left[x_{3}\right]_{x}\right\rangle=\tau\left(\mathbf{x}_{1} \mathbf{x}_{\mathbf{2}} \mathbf{x}_{\mathbf{3}}\right)-\tau\left(\mathbf{x}_{1} \mathbf{x}_{\mathbf{2}}\right)$. Note that the intersection pairing has its values in $\mathcal{M}$.

We pass to the general case. Using the basis $\mathbf{x}_{\mathbf{1}}, \ldots, \mathbf{x}_{\mathbf{n}}$ for the free group $\mathbf{F}_{n}$, we pass to the group ring $\mathbb{Z} \mathbf{F}_{n}$. Let $I$ be the augmentation ideal.

As we have already observed, the homology $H_{1}\left(P_{n}, x, \mathcal{M}_{\text {left }}\right)$ is equal to the first cellular chain module of a wedge of $n$ circles with coefficients in $\mathcal{M}_{\text {left }}$. This is a free right $\mathcal{M}$ module of rank $n$. The tensor product $I \otimes_{\mathbf{F}_{\mathrm{n}}} \mathcal{M}$ is also a free right $\mathcal{M}$ module of rank $n$. The isomorphism of free right $\mathcal{M}$ modules sending the basis element $e_{i}$ of section 2.3 to the element $\left(\mathbf{x}_{i}-1\right) \otimes 1 \in I \otimes \mathcal{M}$ is particularly convenient because then the action action of $B_{n}$ on the left described in section 2.3 corresponds to the action in which an element $\iota \otimes m \in I \otimes \mathcal{M}$ is sent to

$$
\beta(\iota \otimes m)=\beta \iota \beta^{-1} \otimes \beta m
$$


in which the conjugate $\beta \iota \beta^{-1}$ is calculated in the (group ring of the) larger group $\mathbf{B}_{1, n}$. From now on we may as well identify $e_{i}$ with $\left(x_{i}-1\right) \otimes 1$. The homology class of an arc $\gamma$ in $P_{n}$ based at $x$ is determined by its class $\mathbf{g} \in \mathbf{F}_{n}$, by the derivation

$$
d: \mathbf{F}_{n} \rightarrow \oplus e_{i} \mathcal{M}, \quad \mathbf{x}_{\mathbf{i}} \mapsto e_{i}, \quad d(\mathbf{x y})=d(\mathbf{x}) \mathbf{y}+d(\mathbf{y})
$$

The latter rule holds for all $\mathbf{x}, \mathbf{y} \in \mathbf{F}_{\mathbf{n}}$ and is the Leibniz rule for a derivation (a Fox derivative). Note that the right multiplication by $\mathbf{y}$ is by definition the right multiplication using $\tau$ so the formula could more rigorously be written $d(\mathbf{x y})=d(\mathbf{x}) \tau(\mathbf{y})+d(\mathbf{y})$. Note also that the rule implies $d\left(\mathbf{x}^{-1}\right)=-d(\mathbf{x}) \mathbf{x}^{-1}$.

Applying this Fox derivative to the element $\mathbf{w} \in \mathbf{F}_{n}$ which is the homotopy class of the loop $\gamma$ we have

$$
[\gamma]_{x}=d(\mathbf{w}) \in \oplus_{i} e_{i} \mathcal{M}=H_{1}\left(P_{n}, x, \mathcal{M}_{\text {left }}\right) .
$$

For example,

$$
\begin{gathered}
{\left[x_{2} x_{4} x_{2}^{-1}\right]_{x}=d\left(\mathbf{x}_{\mathbf{2}} \mathbf{x}_{\mathbf{4}} \mathbf{x}_{\mathbf{2}}^{-\mathbf{1}}\right)=d\left(\mathbf{x}_{\mathbf{2}}\right) \mathbf{x}_{\mathbf{4}} \mathbf{x}_{\mathbf{2}}^{-\mathbf{1}}+d\left(\mathbf{x}_{\mathbf{4}}\right) \mathbf{x}_{\mathbf{2}}^{-\mathbf{1}}+d\left(\mathbf{x}_{\mathbf{2}}^{-\mathbf{1}}\right)} \\
=e_{2}\left(\mathbf{x}_{\mathbf{4}}-1\right) \mathbf{x}_{\mathbf{2}}^{-\mathbf{1}}+e_{4} \mathbf{x}_{\mathbf{2}}^{-\mathbf{1}}
\end{gathered}
$$

This map $d$ is equivariant under the $\mathbf{B}_{n}$ action by conjugation on $\mathbf{F}_{n}$

$$
d\left(\beta f \beta^{-1}\right)=\beta d(f) \beta^{-1}
$$

Note that in this formula the conjugate $\beta f \beta^{-1}$ can be calculated in $F_{n}$ using the equation 3 while the right side of the equation can be written as $\tau^{+}(\beta) d(f) \tau(\beta)^{-1}$.

The module $H_{1}\left(P_{n}, x, \mathcal{M}_{\text {left }}\right)$ has an $\mathcal{M}$-linear $\mathbf{B}_{n}$ action on the left. This action is the same as the action of $\mathbf{B}_{n}$ on $I \otimes_{\mathbf{F}_{n}} \mathcal{M}$, given by the formula $\beta(\iota \otimes r)=\beta \iota \beta^{-1} \otimes \beta r$, where $\iota \in I, r \in \mathcal{M}$. In terms of the basis $e_{1}, \ldots, e_{n}$ the left $\mathcal{M}$-linear action is given explicitly by:

$$
\sigma_{i} e_{j} r= \begin{cases}e_{j} \tau\left(\sigma_{i}\right) r & \text { if } j \neq i, i+1 \\ e_{i+1} \tau\left(\sigma_{i}\right) r & \text { if } j=i \\ e_{i} \tau\left(\sigma_{i} \mathbf{x}_{\mathbf{i}}\right) r+e_{i+1} \tau\left(\sigma_{i}\right)\left(1-\tau\left(\mathbf{x}_{\mathbf{i}+\mathbf{1}}\right)\right) r & \text { if } j=i+1\end{cases}
$$

while the less-interesting right $B_{n}$ action, which is semilinear with respect to the $B_{n}$ action by conjugation on $\mathcal{M}$, is given

$$
e_{j} r \cdot \sigma_{i}=e_{j} r \tau\left(\sigma_{i}\right) .
$$

Now we repeat everything, this time taking homology with respect to the right action of $\mathbf{B}_{1, n}$ on $\mathcal{M}$, using our basepoint $y$. See Figure 5 (a). In order to describe this module in a way which will later make our intersection pairing simple, we use the involution on $\mathbf{F}_{n}$ which is given by:

$$
\mathrm{x}_{\mathrm{i}} \mapsto \mathrm{y}_{\mathrm{i}}=\mathrm{x}_{1} \mathrm{x}_{2} \ldots \mathrm{x}_{\mathrm{i}}^{-1} \mathrm{x}_{\mathrm{i}-1}^{-1} \ldots \mathrm{x}_{1}^{-1}
$$

We have that $\mathbf{B}_{n}$ acts $\mathcal{M}$-linearly on the right on the left $\mathcal{M}$-module $H_{1}\left(P_{n}, y, \mathcal{M}_{\text {right }}\right)=\bigoplus_{i+1}^{n} \mathcal{M} f_{i}$ where we now take as our basis elements the 
$f_{i}=1 \otimes\left(\mathbf{y}_{\mathbf{i}}-1\right) \in \mathcal{M} \otimes_{\mathbf{F}_{\mathbf{n}}} I$. The right action of $\mathbf{B}_{n}$ is easiest described by giving the action of the inverse of the braid generators:

$$
r f_{j} \sigma_{i}^{-1}= \begin{cases}r \tau\left(\sigma_{i}^{-1}\right) f_{j} & \text { if } j \neq i, i+1 \\ r \tau\left(\mathbf{y}_{\mathbf{i}+\mathbf{1}} \sigma_{i}^{-1}\right) f_{i+1}+r\left(1-\tau\left(\mathbf{y}_{\mathbf{i}}\right)\right) \tau\left(\sigma_{i}^{-1}\right) f_{i} & \text { if } j=i \\ r \tau\left(\sigma_{i}^{-1}\right) f_{i} & j=i+1\end{cases}
$$

We also have the less-interesting left action of $\mathbf{B}_{n}$ on this module which is semilinear for the $B_{n}$ action by conjugation on $\mathcal{M}$, and is given by

$$
\sigma_{i} \cdot r f_{j}=\tau\left(\sigma_{i}\right) r f_{j} .
$$

The homology class of an $\operatorname{arc} \delta$ based at $y$ is given in terms of its homotopy class $g \in \mathbf{F}_{n}$ by the derivation $e(w)$ defined as follows:

$$
e: \mathbf{F}_{n} \rightarrow \oplus \mathcal{M} f_{i}=H_{1}\left(P_{n}, y, \mathcal{M}_{\text {right }}\right), y_{i} \mapsto f_{i}, e(x y)=x e(y)+e(x) .
$$

and we have $[\delta]_{y}=e(g)$, and the derivation $e$ is equivariant in the sense that $e\left(\beta g \beta^{-1}\right)=\beta e(g) \beta^{-1}$.

Now that we have defined two modules, define our $\mathcal{M}$ bilinear pairing $\langle$,

$$
\left(\oplus \mathcal{M} f_{i}\right) \times\left(\oplus e_{i} \mathcal{M}\right) \rightarrow \mathcal{M}
$$

by

$$
\left\langle f_{i}, e_{j}\right\rangle=\left\{\begin{array}{c}
0, i \neq j \\
\tau\left(\mathbf{x}_{\mathbf{1}} \ldots \mathbf{x}_{\mathbf{i}}\right)-\tau\left(\mathbf{x}_{\mathbf{1}} \ldots \mathbf{x}_{\mathbf{i}-\mathbf{1}}\right), i=j
\end{array}\right.
$$

Since the $\tau\left(\mathbf{x}_{\mathbf{i}}\right)-1$ are non-zero-divisors they will become invertible in any flat Artinian fraction ring of $\mathcal{M}$. In other words this becomes a perfect pairing, i.e. $\tau$ becomes non-degenerate. This completes the definition of the pairing.

Remarks on notation: We could have avoided introducing the symbols $e_{i}, f_{i}, d$ and $e$, using instead

$$
e_{i}=\left[x_{i}\right]_{x}, \quad f_{i}=\left[y_{i}\right]_{y}, \quad d(\mathbf{w})=[w]_{x}, \quad e(\mathbf{w})=[w]_{y}
$$

where in the final two expressions $w$ is taken to be a loop based at $x$ or $y$ respectively. However we use the symbols $e_{i}$ and $f_{i}$ to make them look like basis elements in two vector spaces, and using the symbols $d$ and $e$ made them look like derivations. For this reason we chose what some readers may view as an excess of notation.

Proof of (a): To verify our conventions, let us show that the pairing defined in this way really is compatible with the braid action. Thus for $\beta \in \mathbf{B}_{n}$ we need to check that $\left\langle\beta v \beta^{-1}, \beta w \beta^{-1}\right\rangle=\beta\langle v, w\rangle \beta^{-1}$. We may assume that the braid homeomorphism $\beta$ is $\sigma_{i}$ and that our vectors $v$ and $w$ are of the form

$$
v=a f_{i}+b f_{i+1}, \quad w=e_{i} r+e_{i+1} s .
$$

We have

$$
\begin{gathered}
\left\langle\beta v \beta^{-1}, \beta w \beta^{-1}\right\rangle \\
=\left\langle\sigma_{i}\left(a f_{i}+b f_{i+1}\right) \sigma_{i}^{-1}, \sigma_{i}\left(e_{i} r+e_{i+1} s\right) \sigma_{i}^{-1}\right\rangle \\
=\left\langle\sigma_{i} a\left[\mathbf{y}_{\mathbf{i}+\mathbf{1}} \sigma_{i}^{-1} f_{i+1}+\left(1-\mathbf{y}_{\mathbf{i}}\right) \sigma_{i}^{-1} f_{i}\right]+\sigma_{i} b\left[\sigma_{i}^{-1} f_{i+1}\right],\right.
\end{gathered}
$$




$$
\left.\left[e_{i+1} \sigma_{i}\right] r \sigma_{i}^{-1}+\left[e_{i} \sigma_{i} \mathbf{x}_{\mathbf{i}}+e_{i+1} \sigma_{i}\left(1-\mathbf{x}_{\mathbf{i}+\mathbf{1}}\right)\right] s \sigma_{i}^{-1}\right\rangle .
$$

In the formula above the first two expressions in square brackets come from the right side of (14) and the last two expressions in square brackets come from the right side of (12).

Now using definition (3) from section 2.2 and also the definition of the $\mathbf{y}_{\mathbf{i}}$ we know

$$
\begin{gathered}
\mathbf{y}_{\mathbf{i}} \sigma_{i}^{-1}=\sigma_{i}^{-1} \mathbf{y}_{\mathbf{i}+\mathbf{1}} \mathbf{y}_{\mathbf{i}} \mathbf{y}_{\mathbf{i}+\mathbf{1}}^{-\mathbf{1}} \\
\mathbf{y}_{\mathbf{i}+\mathbf{1}} \sigma_{i}^{-1}=\sigma_{i}^{-1} \mathbf{y}_{\mathbf{i}} \\
\sigma_{i} \mathbf{x}_{\mathbf{i}}=\mathbf{x}_{\mathbf{i}+\mathbf{1}} \sigma_{i} \\
\sigma_{i} \mathbf{x}_{\mathbf{i}+\mathbf{1}}=\mathbf{x}_{\mathbf{i}+\mathbf{1}} \mathbf{x}_{\mathbf{i}} \mathbf{x}_{\mathbf{i}+\mathbf{1}}^{-\mathbf{1}} \sigma_{i} .
\end{gathered}
$$

Making these substitutions, and using the notation such that ${ }^{\beta} a$ denotes $\beta a \beta^{-1}$, we obtain

$$
\begin{gathered}
\left\langle\left[{ }^{\sigma_{i}} a\left(1-\mathbf{y}_{\mathbf{i}} \mathbf{y}_{\mathbf{i}+\mathbf{1}} \mathbf{y}_{\mathbf{i}}^{-\mathbf{1}}\right)+{ }^{\sigma_{i}} b\right] f_{i}+{ }^{\sigma_{i}} a \mathbf{y}_{\mathbf{i}} f_{i+1},\right. \\
\left.e_{i+1}\left[\left({ }^{\sigma_{i}} r+\left(1-\mathbf{x}_{\mathbf{i}+\mathbf{1}}^{-\mathbf{1}} \mathbf{x}_{\mathbf{i}} \mathbf{x}_{\mathbf{i}+\mathbf{1}}\right)^{\sigma_{i}} s\right)\right]+e_{i} \mathbf{x}_{\mathbf{i}+\mathbf{1}}{ }^{\sigma_{i}} s\right\rangle
\end{gathered}
$$

and using the definition (17) of the intersection pairing this becomes

$$
\begin{aligned}
& {\left[{ }^{\sigma_{i}} a\left(1-\mathbf{y}_{\mathbf{i}} \mathbf{y}_{\mathbf{i}+\mathbf{1}} \mathbf{y}_{\mathbf{i}}^{-\mathbf{1}}\right)+{ }^{\sigma_{i}} b\right]\left(\mathbf{x}_{\mathbf{1}} \ldots \mathbf{x}_{\mathbf{i}}-\mathbf{x}_{\mathbf{1}} \ldots \mathbf{x}_{\mathbf{i}-\mathbf{1}}\right)\left[\mathbf{x}_{\mathbf{i}+\mathbf{1}}{ }^{\sigma_{i}} s\right] } \\
+ & {\left[{ }^{\sigma_{i}} a \mathbf{y}_{\mathbf{i}}\right]\left(\mathbf{x}_{\mathbf{1}} \ldots \mathbf{x}_{\mathbf{i}+\mathbf{1}}-\mathbf{x}_{\mathbf{1}} \ldots \mathbf{x}_{\mathbf{i}}\right)\left[\left({ }^{\sigma_{i}} r+\left(1-\mathbf{x}_{\mathbf{i}+\mathbf{1}}^{-\mathbf{1}} \mathbf{x}_{\mathbf{i}} \mathbf{x}_{\mathbf{i}+\mathbf{1}}\right)^{\sigma_{i}} s\right)\right] }
\end{aligned}
$$

and when this expression is multiplied out, taking into account $\mathbf{y}_{\mathbf{i}}=\mathbf{x}_{\mathbf{1}} \ldots \mathbf{x}_{\mathbf{i}-\mathbf{1}} \mathbf{x}_{\mathbf{i}}^{-\mathbf{1}} \ldots \mathbf{x}_{\mathbf{1}}^{-\mathbf{1}}$ everything cancels except

$$
{ }^{\sigma_{i}} a\left(\mathbf{x}_{\mathbf{1}} \ldots \mathbf{x}_{\mathbf{i}-\mathbf{1}} \mathbf{x}_{\mathbf{i}+\mathbf{1}}-\mathbf{x}_{\mathbf{1}} \ldots \mathbf{x}_{\mathbf{i}-\mathbf{1}}\right)^{\sigma_{i}} r+{ }^{\sigma_{i}} b\left(\mathbf{x}_{\mathbf{1}} \ldots \mathbf{x}_{\mathbf{i}+\mathbf{1}}-\mathbf{x}_{\mathbf{1}} \ldots \mathbf{x}_{\mathbf{i}} \mathbf{x}_{\mathbf{i}+\mathbf{1}}\right)^{\sigma_{i}} s .
$$

Using $\sigma_{i} \mathbf{x}_{\mathbf{1}} \ldots \mathbf{x}_{\mathbf{i}} \sigma_{i}^{-1}=\mathbf{x}_{\mathbf{1}} \ldots \mathbf{x}_{\mathbf{i}-\mathbf{1}} \mathbf{x}_{\mathbf{i}+\mathbf{1}}$ this becomes

$$
\begin{gathered}
\sigma_{i}\left(a\left(\mathbf{x}_{\mathbf{1}} \ldots \mathbf{x}_{\mathbf{i}}-\mathbf{x}_{\mathbf{1}} \ldots \mathbf{x}_{\mathbf{i}-\mathbf{1}}\right) r+b\left(\mathbf{x}_{\mathbf{1}} \ldots \mathbf{x}_{\mathbf{i}+\mathbf{1}}-\mathbf{x}_{\mathbf{1}} \ldots \mathbf{x}_{\mathbf{i}}\right) s\right) \sigma_{i}^{-1} \\
=\sigma_{i}\left\langle a f_{i}+b f_{i+1}, e_{i} r+e_{i+1} s\right\rangle \sigma_{i}^{-1}=\sigma_{i}\langle v, w\rangle \sigma_{i}^{-1}
\end{gathered}
$$

as desired. This proves statement (a) of the theorem.

Proof of (b): We calculate, using (a).

$$
\begin{aligned}
\langle v \beta, w\rangle & =\beta \beta^{-1}\langle v \beta, w\rangle=\beta\left\langle\beta^{-1} v \beta, \beta^{-1} \beta w \beta^{-1} \beta\right\rangle \\
& =\beta\left[\beta^{-1}\left\langle v, \beta w \beta^{-1}\right\rangle \beta\right]=\langle v, \beta w\rangle
\end{aligned}
$$

Proof of (c): Left to the reader.

Proof of $(\mathbf{d})$ : We can use the anti-involution $*$ on $\mathcal{M}$ to relate the homology class of an arc in either of our two homology groups as follows: Let $e_{i}^{\star} \in \oplus_{i} \mathcal{M} f_{i}$ be defined

$$
e_{i}^{\star}=e\left(\mathbf{x}_{\mathbf{i}}^{-\mathbf{1}}\right)
$$

where $e$ is our derivation. Then $e_{i}^{\star}$ is a basis and we have

$$
\oplus e_{i} \mathcal{M} \rightarrow \oplus \mathcal{M} f_{i}
$$




$$
\sum e_{i} r_{i} \mapsto \sum r_{i}^{\star} e_{i}^{\star}, \quad r_{i} \in \mathcal{M} .
$$

The inverse map sends $f_{i}$ to $f_{i}^{\star}=d\left(\mathbf{y}_{\mathbf{i}}^{-\mathbf{1}}\right)$.

Proof of (e): We are given $v=[w]_{x}$, where $w$ is a simple loop based at $x$.

Every simple $x$-based loop in $P_{n}$ is the image of one of the generators, $\mathbf{x}_{\mathbf{i}}$, of $\mathbf{F}_{n}$ under a braid homeomorphism. Therefore $v=\left[\beta\left(x_{i}\right)\right]_{x}=\beta\left[x_{i}\right]_{x} \beta^{-1}=\beta e_{i} \beta^{-1}$ for some $\beta \in \mathbf{B}_{n}$. (We could even take $i=1$.) Then we have

$$
\left\langle v^{*}, v\right\rangle=\left\langle\beta e_{i}^{*} \beta^{-1}, \beta e_{i} \beta^{-1}\right\rangle=\beta\left\langle e_{i}^{\star}, e_{i}\right\rangle \beta^{-1} .
$$

Now we have $e_{i}^{\star}=e\left(\mathbf{x}_{\mathbf{i}}^{-\mathbf{1}}\right)$ where $e$ is the derivation sending $\mathbf{y}_{\mathbf{i}}$ to $f_{i}$. Writing

$$
\mathrm{x}_{\mathrm{i}}^{-1}=\mathrm{y}_{1} \mathrm{y}_{2} \ldots \mathrm{y}_{\mathrm{i}} \mathrm{y}_{\mathrm{i}-1}^{-1} \ldots \mathrm{y}_{1}^{-1}
$$

we expand using the rule $e(\mathbf{a b})=\mathbf{a} d(\mathbf{b})+e(\mathbf{a})$. We find that the term involving $f_{i}$ is $\mathbf{y}_{1} \mathbf{y}_{2} \ldots \mathbf{y}_{\mathbf{i}-1} f_{i}$ and therefore

$$
\begin{gathered}
\left\langle e_{i}^{\star}, e_{i}\right\rangle=\mathbf{y}_{\mathbf{1}} \ldots \mathbf{y}_{\mathbf{i}-\mathbf{1}}\left\langle f_{i}, e_{i}\right\rangle \\
=\tau\left(\mathbf{y}_{\mathbf{1}} \ldots \mathbf{y}_{\mathbf{i}-\mathbf{1}}\right)\left(\tau\left(\mathbf{x}_{\mathbf{1}} \ldots \mathbf{x}_{\mathbf{i}}\right)-\tau\left(\mathbf{x}_{\mathbf{1}} \ldots \mathbf{x}_{\mathbf{i}-\mathbf{1}}\right)\right)
\end{gathered}
$$

which simplifies to $\tau\left(\mathbf{x}_{\mathbf{i}}\right)-1$. Then

$$
\left\langle v^{\star}, v\right\rangle=\beta^{-1}\left(\tau\left(\mathbf{x}_{\mathbf{i}}\right)-1\right) \beta=\tau\left(\beta\left(\mathbf{x}_{\mathbf{i}}\right)\right)-1=\tau(\mathbf{w})-1 .
$$

Proof of (f): This part of the theorem relates the intersection pairing with the the geometric intersections of arcs on surfaces. Throughout this proof we are assuming that $\tau^{+}$is faithful. Therefore we needn't make any distinction between a braid and its associated matrix. We need a braid element $\omega$ in the kernel of the representation $\tau$. (This is where we use the hypothesis that $\tau$ is non-faithful for some $r$.) Suppose our element $\omega$ is a braid on $r$ strands. Say $\delta$ is a loop based at $y$ which encircles only $q_{i}$ and $\gamma$ is a loop based at $x$ which encircles only $q_{j}$ and that $\left\langle[\delta]_{y},[\gamma]_{x}\right\rangle=0$. Recall $y_{k}$ is the arc based at $y$ whose homotopy class is $\mathbf{y}_{\mathbf{k}}$. We may find a braid $\kappa$ such that $\kappa(\delta)=y_{k}$ for some number $k$ and we have

$$
\left\langle[\delta]_{y},[\gamma]_{x}\right\rangle=\kappa\left\langle f_{k}, \kappa^{-1}[\gamma] \kappa\right\rangle \kappa^{-1}=\kappa\left\langle f_{k},\left[\kappa^{-1} \gamma\right]\right\rangle \kappa^{-1} .
$$

Let us re-name our arcs, giving $\kappa^{-1} \gamma$ the name $\mu$. Now we are in the situation where

$$
\left\langle f_{k},[\mu]_{x}\right\rangle=0
$$

and we wish to show $\mu$ can be homotoped to not meet the arc $y_{k}$. First note that $\mu$ does not encircle $q_{k}$ because if we expand $[\mu]$ in the form $e_{1} c_{1}+\ldots+e_{n} c_{n}$ then $0=\left\langle f_{k}, \mu\right\rangle=\tau\left(\mathbf{x}_{\mathbf{1}} \ldots \mathbf{x}_{\mathbf{i}-\mathbf{1}}\right)\left(\tau\left(\mathbf{x}_{\mathbf{i}}\right)-1\right) c_{k}$ forces $c_{k}$ to be zero, because $\tau\left(\mathbf{x}_{\mathbf{i}}\right)-1$ is a nonzero divisor. But $c_{k}$ would be a sum of elements of the form $\pm \tau(\mathbf{w})$ for $\mathbf{w} \in F_{n}$ whose coefficient sum adds to the integer +1 if $\mu$ encircled $q_{k}$ and such a sum is congruent modulo the $\tau\left(\mathbf{x}_{\mathbf{i}}\right)-1$ to the element $1 \in \mathcal{M}$. By hypothesis the ideal generated by the $\tau\left(\mathbf{x}_{\mathbf{i}}\right)-1$ in the appropriate subring is not the unit ideal so this is impossible. By enlarging $n$, in effect adding $r-1$ new points numbered $q_{k+1}, \ldots, q_{k+r-1}$ to our set $Q_{n}$ and renumbering, we may assume $\mu$ does not meet the arcs $y_{k+1}, \ldots, y_{k+r-1}$ based at $y$ whose homotopy classes are $\mathbf{y}_{\mathbf{k}+\mathbf{1}}, \ldots, \mathbf{y}_{\mathbf{k}+\mathbf{r}-\mathbf{1}}$, so we may assume

$$
\left\langle f_{i},[\mu]_{x}\right\rangle=0
$$


for $i=k+1, \ldots, k+r-1$. By further enlarging $\left\{q_{i}\right\}$, adding new points $q_{1}, \ldots, q_{r}$ and renumbering, we may assume $\mu$ also does not meet the arcs $y_{1}, \ldots, y_{r}$ based at $y$ whose homotopy classes are $\mathbf{y}_{\mathbf{1}}, \ldots, \mathbf{y}_{\mathbf{r}}$ so we also have

$$
\left\langle f_{i},[\mu]_{x}\right\rangle=0
$$

for the values $i=1,2, \ldots, r$ as well. In all, we have $\left\langle f_{i},[\mu]_{x}\right\rangle=0$ for $i=1,2,3, \ldots, r, k, k+1, \ldots, k+r-1$.

We can let $\sigma$ be a second copy of the braid $\omega$ but which acts on strands $k, k+1, \ldots, k+r-1$ so that the left action of $\sigma$ on only the basis elements $e_{k}, \ldots, e_{k+r-1}$ is nontrivial, and the action of $\sigma$ on $\mathcal{M}$ is trivial.

Let $x_{r}$ as usual be the simple arc based at $x$ which encircles just $q_{r}$, whose homotopy class is $\mathbf{x}_{\mathbf{r}}$. Let $\alpha$ be a braid which does not affect strands $1,2, \ldots, \mathrm{r}-1$ such that $\alpha\left(x_{r}\right)=\mu$. We claim that the conjugate braid $\alpha^{-1} \sigma \alpha$ fixes the vectors $e_{1}, . ., e_{r} \in \oplus e_{i} \mathcal{M}$. It clearly fixes $e_{1}, \ldots, e_{r-1}$ since the braid does not involve the corresponding strands. As for $e_{r}$ write

$$
\alpha e_{r}=e_{1} c_{1}+\ldots+e_{n} c_{n} .
$$

The coefficients $c_{i}$ which are zero are the same as the ones which are zero if instead we calculated $\alpha e_{r} \alpha^{-1}$. Thus we calculate

$$
\left\langle f_{i}, \alpha e_{r} \alpha^{-1}\right\rangle=\left\langle f_{i}, \alpha\left[x_{r}\right] \alpha^{-1}\right\rangle=\left\langle f_{i},\left[\alpha\left(x_{r}\right)\right]\right\rangle=\left\langle f_{i},[\mu]\right\rangle
$$

which is zero for $i=k, \ldots, k+r-1$. This proves $c_{i}=0$ for $i=k, k+1, \ldots, k+r-1$. Since the braid $\sigma$ is in the kernel of the action on $\mathcal{M}$ it acts only on coefficients which correspond to the strands it acts on, ie only on coefficients $c_{k}, c_{k}+1, \ldots, c_{k+r-1}$ of $\alpha e_{r}$, and yet these coefficients are all zero. This shows $\sigma$ fixes $\alpha e_{r}$ so $\alpha^{-1} \sigma \alpha$ fixes $e_{r}$ as claimed.

Now one sees that the commutator $\left[\alpha^{-1} \sigma \alpha, \omega\right]$ also fixes the vectors $e_{1}, \ldots, e_{r}$. This is because $\omega$ preserves $\oplus_{i=1}^{r} e_{i} \mathcal{M}$ and $\alpha^{-1} \sigma \alpha$ has no effect on this module. Now write

$$
\beta=\alpha^{-1} \sigma \alpha
$$

and let us calculate the action of a commutator

$$
\left[\beta^{s}, \omega^{t}\right]
$$

on the whole module, for numbers $s$ and $t$. We already know $e_{1}, \ldots, e_{r}$ are fixed. Thus let $i \geq r+1$ and let us calculate. Write

$$
\beta^{-s} e_{i}=e_{r} a_{r}+\ldots+e_{n} a_{n}
$$

and

$$
\omega^{t} e_{r}=e_{1} d_{1}+\ldots+e_{r} d_{r}
$$

Then

$$
\begin{gathered}
\beta^{s} \omega^{t} \beta^{-s} w^{-t} e_{i}=\beta^{s} \omega^{t} \beta^{-s} e_{i}=\beta^{s} \omega^{t}\left(e_{r} a_{r}+\ldots+e_{n} a_{n}\right) \\
=\beta^{s}\left(e_{1} d_{1} a_{r}+\ldots+e_{r} d_{r} a_{r}+e_{r+1} a_{r+1}+\ldots+e_{n} a_{n}\right) \\
=\beta^{s}\left(e_{1} d_{1} a_{r}+\ldots+e_{r} d_{r} a_{r}-e_{r} a_{r}+\beta^{-s} e_{i}\right)
\end{gathered}
$$




$$
=e_{1} d_{1} a_{r}+\ldots+e_{r}\left(d_{r}-1\right) a_{r}+e_{i} .
$$

We see that the action of the commutator adds to the $i^{\prime} t h$ basis vector only some multiples of basis vectors $e_{1}, \ldots, e_{r}$. In other words, the action of the commutator lies in an abelian group of elementary matrices with entries in $\mathcal{M}$. It follows that for different values of $s$ and $t$ the commutators all commute. In other words,

$$
1=\left[\left[\beta^{s}, \omega^{t}\right],\left[\beta^{s^{\prime}}, \omega^{t^{\prime}}\right]\right]
$$

for all $s, t, s^{\prime}, t^{\prime}$.

Any such relation (for any given values of these numbers) continues to hold if we substitute $w$ and $b$ for any powers, and it follows from McCarthy's Tits alternative for braid groups 22 that $\beta$ and $\omega$ commute.

Now, we could have chosen $\omega$ pseudo-Anosov, and so $\beta$ and $\omega$ commuting that means $\beta$ is a braid which does not involve the $r^{\prime}$ th braid generator $\sigma_{r}$. This means that $\beta$ (which we already know does not involve the braid generators $\sigma_{1}, \ldots, \sigma_{r-1}$ ) also does not involve $\sigma_{r}$. In other words, $\alpha^{-1} \sigma \alpha$ preserves the homotopy type of $x_{r}$. Then $\sigma$ preserves the homotopy type of $\alpha\left(x_{r}\right)=\mu$ and again choosing $\sigma$ pseudo-Anosov on strands $k, \ldots, k+r-1$ we see this means $\mu$ does not meet the arc with homotopy class $y_{k}$, as needed. This completes the proof of (f) and so also of Theorem 1. ॥

\section{Detecting reducing loops}

We are now ready to make the connection between the existence of a reducing loop in a closed braid $\tilde{\beta}$ and our intersection pairing on arcs in the n-times punctured plane $P_{n}=P-Q_{n}$, under the action of $\beta \in \mathbf{B}_{n}$ :

Theorem $2 A$ braid $\beta \in \mathbf{B}_{n}$ is conjugate to a positively (respectively negatively) reducible braid if and only if there is a simple homology class $v \in H_{1}\left(P_{n}, x, \mathcal{M}_{\text {left }}\right)$ such that $\left\langle v^{\star}, \beta v \beta^{-1}\right\rangle=0\left(\right.$ resp. $\left.\left\langle\beta v^{\star} \beta^{-1}, v\right\rangle=0\right)$.

Proof: We will treat the positively reducible case, the negative case being similar. Suppose we have a simple homology class $v$ such that $\left\langle v^{\star}, \beta v \beta^{-1}\right\rangle=0$. The homology class $v$, being assumed simple, is represented by an arc $\eta$ based at $x$ encircling some $q_{i}$. We know that the homology class $v=[\eta]_{x}$ satisfies

$$
0=\left\langle v^{\star}, \beta v \beta^{-1}\right\rangle=\left\langle[\eta]^{\star},[\beta \eta]\right\rangle .
$$

Suppose that $\eta$ encircles $q_{i}$ and $\beta \eta$ encircles $q_{j}$. We wish to apply Theorem 1 Let $\omega$ be a loop based at $y$ which represents the same element of $\pi_{1}\left(P_{n}, y\right)$ as $\eta$. Then we have $0=\left\langle[\omega]_{y},[\beta \eta]_{x}\right\rangle$ and the theorem says we can homotop the two loops $\omega$ and $\beta(\eta)$ apart while fixing both baseoints. Another way of describing the same information is that if we had had connected the basepoint $x$ to $q_{i}$ by an arc $\gamma$ entirely contained within the closed loop $\eta$ then $\gamma \cup \beta(\gamma)$ could be be homotoped fixing both endpoints $q_{i}$ and $q_{j}$ so that the union is a simple arc from $q_{i}$ to $q_{j}$ passing through the basepoint $x$. Near the basepoint the orientation of this arc is clockwise (in the direction from $y$ to $x$ ) and this is why it is a positive reducing move. Moreover, rather than modify $\beta \gamma$ by a homotopy, we can choose the braid isotopy so that $\gamma$ and $\beta \gamma$ actually meet only at the 
basepoint. Finally, it helps to move the basepoint away from the axis $A$ slightly, to a a new point $x^{\prime}$ nearby in the interior of $P$. We will be done if we can prove the following lemma, which actually shows how the reducing move may be carried out. Note that the lemma does not distinguish between positive and negative reducing moves.

Lemma 4.1 Let $\beta$ be a braid homeomorphism. Let $x^{\prime} \in P$ be a basepoint which is outside the disc where $\beta$ acts. Let $\gamma$ be a simple arc in $P$ which joins $x^{\prime}$ to $q_{i}$. Then there is a reducing move replacing the $i^{\text {th }}$ strand of the closed braid $\tilde{\beta}$ by an arc in the plane $P$ which joins $q_{i}$ to $\beta\left(q_{i}\right)$, if and only if $\gamma$ can be homotoped to intersect $\beta(\gamma)$ precisely in the point $\left\{x^{\prime}\right\}$

Proof: Suppose $\gamma$ can be homotoped to meet $\beta \gamma$ only at $x^{\prime}$. Composing the $\beta$ with a suitable homeomorphism we may assume $\gamma$ actually meets $\beta(\gamma)$ only at $x^{\prime}$. Recall the map $\mathbf{H}: P \times[0,1] \rightarrow \mathbb{R}^{3}$ from section 2.1 Consider the image under $\mathbf{H}$ of the square $\gamma \times[0,1]$. The square has four edges:

$$
\gamma \times\{0\}, \gamma \times\{1\},\{x\} \times[0,1] \text { and }\left\{q_{i}\right\} \times[0,1] .
$$

The map $\mathbf{H}$ fixes the arc $\gamma=\gamma \times\{0\}$, sends $\gamma \times 1$ to $\beta(\gamma)$, sends $\left\{x^{\prime}\right\} \times[0,1]$ to a small homotopically trivial loop about the axis $A$ and sends $q_{i} \times[0,1]$ to the $i^{t h}$ strand of the closed braid associated to $\beta$ in $\mathbb{R}^{3}$. The square describes a homotopy by which the $i^{\text {th }}$ strand can be homotoped, fixing both ends (so the rest of the braid is not affected) to the composite of three paths: $\beta(\gamma)$, a small homotopically trivial closed loop about the axis $A$, and $\gamma$. You can cut out the homotopically trivial closed loop without affecting the homotopy type of the link in $\mathbb{R}^{3}$, and what remains is the original closed braid in $\mathbb{R}^{3}$ with its $i^{\text {th }}$ strand replaced by the $\operatorname{arc} \gamma \cup \beta(\gamma)$ in the half plane $P$. This is the desired reducing move. The converse should also be clear, i.e. if a reducing move replaces the $i^{t h}$ strand by an arc in $P$ joining $q_{i}$ to $\beta\left(q_{i}\right)=q_{j}$, then the arc can always be deformed, in the complement of $Q_{n}$, to an arc of the form $\gamma \cup \beta(\gamma)$, where $\gamma$ runs from $x^{\prime}$ to $q_{i}$. This completes the proof of the Lemma, and so also of the Theorem. $\|$

Example: We use Theorem 2 to show that the braid $\beta_{2}=\sigma_{2}^{-2} \sigma_{1}^{-1} \sigma_{2}^{-1} \sigma_{3}^{-1} \sigma_{2}^{3} \sigma_{1} \sigma_{2} \sigma_{3}$ which is illustrated in Figure पa) has a negative reducing loop. It will be clear later why we have named this braid $\beta_{2}$. We do this by exhibiting a simple homology class $v \in H_{1}\left(P_{n}, x, \mathcal{M}_{\text {left }}\right)$ such that $\left\langle\beta_{2} v^{\star} \beta_{2}^{-1}, v\right\rangle=0$. We claim that we can take $v=\left[x_{3}\right]_{x}=e_{3}$. To see this, use the action given in (12) to verify that $\beta_{2} e_{3}=e_{1} \tau\left(\beta_{2}\right)=e_{1} \beta_{2}$, so that $\left\langle\beta_{2} e_{3}^{\star} \beta_{2}^{-1}, e_{3}\right\rangle=\left\langle f_{1}, e_{3}\right\rangle$, which is indeed zero.

Remark 1: The algebraic action of the braid $\beta_{2}=\sigma_{2}^{-2} \sigma_{1}^{-1} \sigma_{2}^{-1} \sigma_{3}^{-1} \sigma_{2}^{3} \sigma_{1} \sigma_{2} \sigma_{3}$ on $\pi_{1}\left(\mathbf{F}_{\mathbf{n}}\right)$ was given in (3). This algebraic action is right to left (functional notation), so that $\beta_{2} \mathbf{x}_{\mathbf{3}} \beta_{2}^{-1}=\mathbf{x}_{\mathbf{1}}$. This is opposite to the geometric action which is we depicted earlier, in Figure 1 (d). The arc we called $\delta^{\prime}$ determines the loop $x_{1}$. We obtain from it the arc we call $\beta\left(\delta^{\prime}\right)$ (which determines $x_{3}$ ) by pushing it one full turn around the oriented braid axis, keeping its endpoint on $\tilde{K}$. Thus the algebraic action is opposite in sense to the geometric action.

Remark 2: It should be clear to the reader, from this example, that the question of enumerating simple homology classes $e_{1} a_{1}+e_{2} a_{2}+\cdots+e_{n} a_{n}$ in 
$H_{1}\left(P_{n}, x, \mathcal{M}_{\text {left }}\right)$ is the key to applying Theorem 2 to obtain an algorithm for recognizing reducing loops. See the discussion on open problems at the end of the paper.

\section{Detecting exchange moves}

Recall the conjugacy class of a closed braid $\tilde{K}$ is said to admit an exchange move if it contains a representative of the form $P \sigma_{n-1} Q \sigma_{n-1}^{-1}$.

Theorem $3 A$ braid $\beta \in \mathbf{B}_{n}$ admits an exchange move if and only if there are simple homology classes $v, w \in H_{1}\left(P_{n}, x, \mathcal{M}_{\text {left }}\right)$ such that $\left\langle v^{\star}, w\right\rangle=0$ and $\left\langle v^{\star}, \beta w \beta^{-1}\right\rangle=0$.

Observe that if, instead $v$ and $w$ are such that $\left\langle v^{*}, w\right\rangle=\left\langle\beta v^{*} \beta^{-1}, w\right\rangle=0$ then 'conjugating' the second formula by $\beta$ gives $\left\langle v^{*}, w\right\rangle=\left\langle v^{*}, \beta^{-1} w \beta\right\rangle=0$. It follows that $v$ and $\beta^{-1} w \beta$ are two simple classes satisfying the hypothesis, so either version implies the other.

Proof: The proof of Theorem 3 is very closely related to the proof of Theorem 2] Assume that

$$
\left\langle v^{\star}, w\right\rangle=\left\langle v^{\star}, \beta w \beta^{-1}\right\rangle=0 .
$$

We must obtain a pair of $\operatorname{arcs} \gamma, \delta$ in $P_{n}$, with $\gamma$ based at $y$ and $\delta$ based at $x$ such that $\gamma$ meets neither $\delta$ nor $\beta(\delta)$. We have $\gamma$ ending at say $q_{i}$ and $\delta$ ending at $q_{j}$. We may move the basepoints $y$ and $x$ in $P$ a little away from the axis but still outside the range where $H$ acts.

We may assume that $H(p, t)=p$ for $0 \leq t \leq 1 / 2$, and now we do two homotopies. By doing a homotopy within the square $\mathbf{H}(\gamma \times[0,1 / 2])$, we replace strand $i$ for $0 \leq t \leq 1 / 2$ by the image under $\mathbf{H}$ of

$$
(\gamma \times\{0\}) \cup(\{q\} \times[0,1 / 2]) \cup(\gamma \times\{1 / 2\}) .
$$

Similarly, we replace strand $j$ for $1 / 2 \leq t \leq 1$ by the image under $\mathbf{H}$ of

$$
(\tau \times\{1 / 2\}) \cup(\{r\} \times[1 / 2,1]) \cup(\beta(\tau) \times\{1\}) .
$$

The homotopy in the second case takes place within the square $\mathbf{H}(\tau \times[1 / 2,1])$. Now our braid contains a pair of small semicircles about the axis $A$, and we can 'interchange' the positions of the semicircles in $\mathbb{R}^{3}$ without affecting the type of the closed braid. The homotopies are then reversed and the result is an exchange move.

Remark 3. An explicit way to calculate the new braid after the exchange has taken place is as follows: Assume that $\left\langle v^{*}, w\right\rangle=0$ and $\left\langle v^{*}, \beta w \beta^{-1}\right\rangle=0$. Choose a braid $\psi$ such that $v=\left[\psi x_{n-1}\right]_{x}$ and $w=\left[\psi x_{n}\right]_{x}$. Choose a braid $\phi$ such that $v=\left[\phi x_{n-1}\right]_{x}$ and $\beta w \beta^{-1}=\left[\phi x_{n}\right]_{x}$. Then $\beta$ will be replaced by $\phi \sigma_{n-1}^{-2} \phi^{-1} \beta \psi \sigma_{n-1}^{2} \psi^{-1}$.

Example: An example is the closed 4-braid $\beta=\left(\sigma_{2}^{-2} \sigma_{1} \sigma_{2}^{-1}\right)\left(\sigma_{3}\right)\left(\sigma_{2}^{3} \sigma_{1}^{-1} \sigma_{2}\right)\left(\sigma_{3}^{-1}\right)=P \sigma_{3} Q \sigma_{3}^{-1}$. See Figure 6. It's an interesting example because the associated closed braid represents the unknot, 
but the closed braid does not have a reducing loop. However after two exchange moves (see the right sketch) it is changed to the braid $K$ which we showed earlier in Figure 1 That braid has a positive reducing loop. The example was discovered by Hugh Morton [26].

We now show that the two exchange moves are detected by the algebra:

- The first exchange move: We need to find simple homology classes

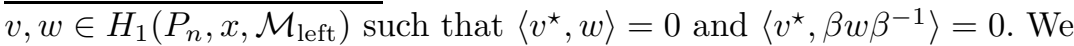
claim that $v=\left[x_{1}\right]_{x}=e_{1}$ and

$$
\begin{gathered}
w=\left[x_{2} x_{4} x_{2}^{-1}\right]_{x}=d\left(\mathbf{x}_{\mathbf{2}} \mathbf{x}_{\mathbf{4}} \mathbf{x}_{\mathbf{2}}^{-\mathbf{1}}\right)= \\
d\left(\mathbf{x}_{\mathbf{2}}\right) \mathbf{x}_{\mathbf{4}} \mathbf{x}_{\mathbf{2}}^{-\mathbf{1}}+d\left(\mathbf{x}_{\mathbf{4}}\right) \mathbf{x}_{\mathbf{2}}^{-\mathbf{1}}+d\left(\mathbf{x}_{\mathbf{2}}^{-\mathbf{1}}\right)=e_{2}\left(\mathbf{x}_{\mathbf{4}}-1\right) \mathbf{x}_{\mathbf{2}}^{-\mathbf{1}}+e_{4} \mathbf{x}_{\mathbf{2}}^{-\mathbf{1}}
\end{gathered}
$$

do the job. We have $\left\langle v^{\star}, w\right\rangle=\left\langle e_{1}^{\star}, w\right\rangle=\left\langle f_{1}, w\right\rangle=0$ because the coefficient of $e_{1}$ in the expression for $w$ is zero. This is the first part of the test for the first exchange move. Next, a calculation gives

$$
\beta w \beta^{-1}=e_{2}\left(\mathbf{x}_{\mathbf{3}} \mathbf{x}_{\mathbf{2}} \mathbf{x}_{\mathbf{3}}^{-1} \mathbf{x}_{\mathbf{2}}^{-1}+\mathbf{x}_{\mathbf{3}}^{-1} \mathbf{x}_{\mathbf{2}}^{-1}-\mathbf{x}_{\mathbf{2}}^{-1}\right)+e_{3}\left(\mathbf{x}_{\mathbf{2}} \mathbf{x}_{\mathbf{3}}^{-1} \mathbf{x}_{\mathbf{2}}^{-1}-\mathbf{x}_{\mathbf{3}}^{-1} \mathbf{x}_{\mathbf{2}}^{-1}\right)
$$

and this does not involve $e_{1}$ so that

$$
\left\langle v^{*}, \beta w \beta^{-1}\right\rangle=\left\langle e_{1}^{*}, \beta w \beta^{-1}\right\rangle=\left\langle f_{1}, \beta w \beta^{-1}\right\rangle=0 .
$$

This shows that there is an exchange move relating the homology classes $v$ and $w$. If one calculates the exchange move by the remark above, one sees that the braid $\beta$ has been changed to $\beta_{1}=\sigma_{2}^{-2} \sigma_{1}^{-1} \sigma_{2}^{-1} \sigma_{3} \sigma_{2}^{3} \sigma_{1} \sigma_{2} \sigma_{3}^{-1}$

- The second exchange move: Start with the braid $\beta_{1}$. We consider the homology classes $v=\left[x_{1} x_{2} x_{3} x_{4} x_{3}^{-1} x_{2}^{-1} x_{1}^{-1}\right]_{x}$ and $w=\left[x_{1} x_{2} x_{3} x_{2}^{-1} x_{1}^{-1}\right]_{x}$ Note that $v^{*}=f_{4}$ and the rule for applying the derivation $d$ gives

$$
\begin{gathered}
w=d\left(\mathbf{x}_{\mathbf{1}} \mathbf{x}_{\mathbf{2}} \mathbf{x}_{\mathbf{3}} \mathbf{x}_{\mathbf{2}}^{-\mathbf{1}} \mathbf{x}_{\mathbf{1}}^{-\mathbf{1}}\right)= \\
e_{1}\left(\mathbf{x}_{\mathbf{2}} \mathbf{x}_{\mathbf{3}} \mathbf{x}_{\mathbf{2}}^{-\mathbf{1}} \mathbf{x}_{\mathbf{1}}^{-\mathbf{1}}-\mathbf{x}_{\mathbf{1}}^{-\mathbf{1}}\right)+e_{2}\left(\mathbf{x}_{\mathbf{3}} \mathbf{x}_{\mathbf{2}}^{-\mathbf{1}} \mathbf{x}_{\mathbf{1}}^{-\mathbf{1}}-\mathbf{x}_{\mathbf{2}}^{-\mathbf{1}} \mathbf{x}_{\mathbf{1}}^{-\mathbf{1}}\right)+e_{3} \mathbf{x}_{\mathbf{2}}^{-\mathbf{1}} \mathbf{x}_{\mathbf{1}}^{-\mathbf{1}}
\end{gathered}
$$

Since the coefficient of $e_{4}$ in $w$ is zero, whereas $v^{*}=f_{4}$, we have $\left\langle v^{*}, w\right\rangle=0$.

Now we calculate $\left\langle v^{*}, \beta_{1} w \beta_{1}^{-1}\right\rangle$ and this can be done most easily by noting that in the action of the braid automorphism $\beta_{1}$ we have

$$
\beta_{1}\left(\mathbf{x}_{1} \mathbf{x}_{2} \mathbf{x}_{3} \mathbf{x}_{2}^{-1} \mathbf{x}_{1}^{-1}\right)=\mathbf{x}_{1} \mathbf{x}_{2} \mathbf{x}_{3} \mathbf{x}_{1} \mathbf{x}_{3}^{-1} \mathbf{x}_{2}^{-1} \mathbf{x}_{1}^{-1}
$$

so that

$$
\beta_{1} w \beta_{1}^{-1}=\left[x_{1} x_{2} x_{3} x_{1} x_{3}^{-1} x_{2}^{-1} x_{1}^{-1}\right]_{x} .
$$

When we calculate this using the derivation rule we see that no basis element $e_{4}$ occurs, because the word does not involve $x_{4}$ at all. Thus

$$
\left\langle v^{*}, \beta_{1} w \beta_{1}^{-1}\right\rangle=\left\langle f_{4}, \beta_{1} w \beta_{1}^{-1}\right\rangle=0 .
$$

When we calculate the new braid we obtain a braid $\beta_{2}$ which differs from $\beta_{1}$ in that the $\sigma_{3}$ crossings have been reversed. The result $\beta_{2}$ is the braid in figure 1 , and as we have already seen in the example in section 4 , this braid satisfies $\left\langle\beta_{2} e_{3}^{*} \beta_{2}^{-1}, e_{3}\right\rangle=0$ so it admits a reducing move to a braid on three strands only. 
(1)

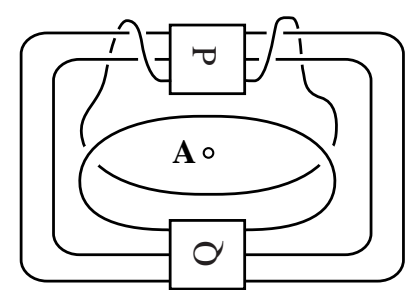

exchange move and braid isotopy reduce braid length

(2)
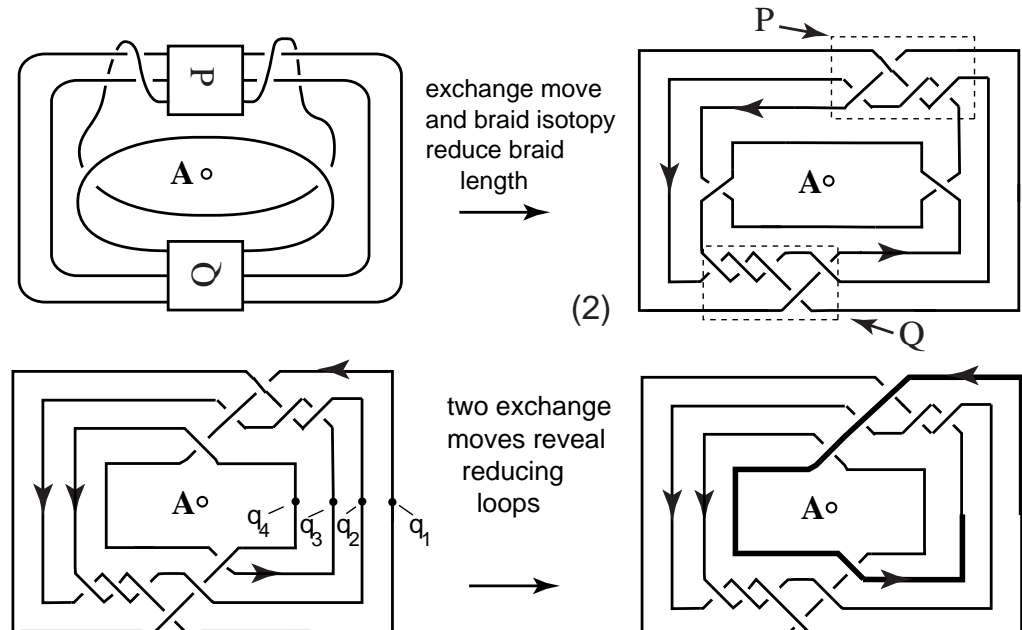

(3) $\sigma_{2}^{-2} \sigma_{1} \sigma_{2}^{-1} \sigma_{3} \sigma_{2}^{3} \sigma_{1}^{-1} \sigma_{2} \sigma_{3}^{-1}=4$ - braid

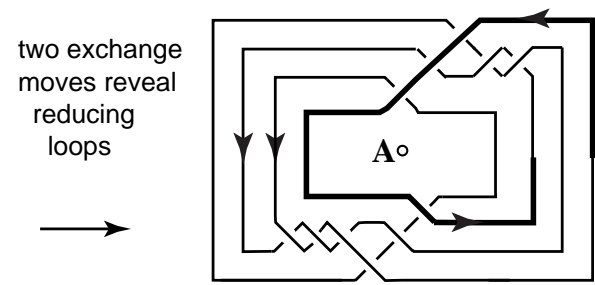

(4) $\sigma_{2}^{-2} \sigma_{1}^{-1} \sigma_{2}^{-1} \sigma_{3}^{-1} \sigma_{2}^{3} \sigma_{1} \sigma_{2} \sigma_{3}=4$-braid

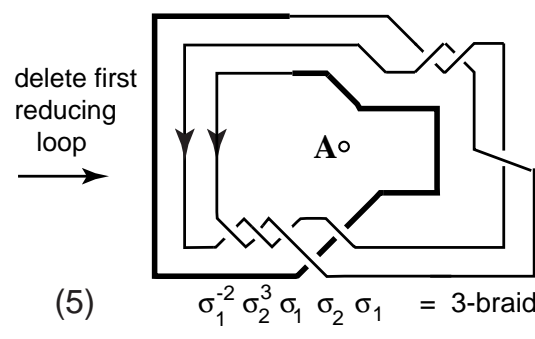

delete second reducing loop
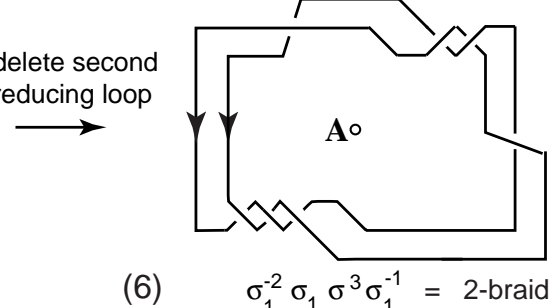

(6)

$\sigma_{1}^{-2} \sigma_{1} \sigma^{3} \sigma_{1}^{-1}=2$-braid

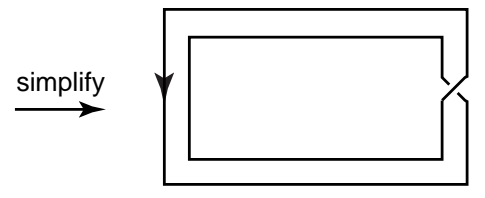

(7)

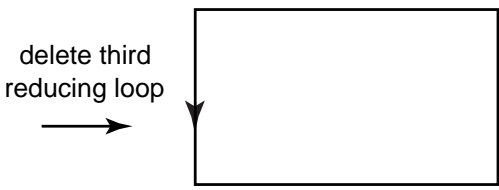

(8)

1-braid

Figure 6: In this closed braid representative of the unknot two exchange moves are needed before the reducing loop on the right (which is the same as that in the left sketch in Figure 1) is revealed. 


\section{Detecting intersections via matrix entries}

In this section we'll explain exactly what it means for a matrix entry to be zero, and look at the corresponding very special cases of the reduction and exchange move conditions. These are weaker than Theorems 2 and 3 Using the notation set up above, with the basis $e_{1}, \ldots, e_{n}$ of $\oplus e_{i} \mathcal{M}$ and basis $f_{1}, \ldots, f_{n}$ of $\oplus \mathcal{M} f_{i}$, recall the intersection pairing which we defined in Equation (17). Let $t_{i} \in \mathcal{M}$ be the element

$$
t_{i}=\tau\left(\mathbf{x}_{\mathbf{1}} \ldots \mathbf{x}_{\mathbf{i}}\right)-\tau\left(\mathbf{x}_{\mathbf{1}} \ldots \mathbf{x}_{\mathbf{i}-\mathbf{1}}\right)
$$

(Here we use the representation $\tau: \mathbf{B}_{1, n} \rightarrow \mathcal{M}$ as a method to view any element of $\mathbf{B}_{n}$ or $\mathbf{F}_{n}$ as belonging to $\left.\mathcal{M}\right)$.

If $\beta \in \mathbf{B}_{n}$ is a braid then the image of $\beta$ under our matrix representation of $\mathbf{B}_{n}$ is the matrix $\left(r_{i j}\right) \in G L_{n}(\mathcal{M})$ defined by:

$$
\beta e_{j}=e_{1} r_{1 j}+\ldots+e_{n} r_{n j} .
$$

First we shall explain exactly what it means for a matrix entry $r_{i j}$ to be zero. Recall if we take $\mathrm{M}$ to be the Magnus matrix ring then each element of $\mathcal{M}$ is an $n+1$ by $n+1$ matrix with entries in $\mathbb{Z}\left[q, q^{-1}, t, t^{-1}\right]$, so that $\left(r_{i j}\right)$ can be thought of as a matrix whose entries are $n+1$ by $n+1$ blocks.

Lemma 6.1 The matrix entry $r_{i j}$ is zero if and only if $\beta=b c$ where strand $i$ at the top only makes undercrossings in $b$ and strand $j$ at the bottom only makes overcrossings in $c$, and these are two distinct strands. In other words if and only if $\beta=\sigma_{i+1} \sigma_{i+2} \ldots \sigma_{n-1} P \sigma_{n-1}^{-1} Q \sigma_{n-1} \sigma_{n-2} \ldots \sigma_{j+1}$ for some $P, Q \in \mathbf{B}_{n-1}$.

Proof: We calculate

$$
\left\langle f_{i}, \beta e_{j}\right\rangle=t_{i} r_{i j}
$$

If $r_{i j}=0$ this means that

$$
\left\langle f_{i}, \beta e_{j}\right\rangle=0
$$

Since $t_{i}$ is a nonzero divisor it follows that (21) is equivalent to $r_{i j}=0$.

As usual let $x_{i}$ be the loop at the basepoint $x$ which encircles $q_{j}$ in the simplest possible way, so that $e_{j}=\left[x_{j}\right]_{x}$ is the homology class of $x_{j}$. From the formulas given earlier we have:

$$
\left[\beta x_{j}\right]_{x}=\beta\left[x_{j}\right]_{x} \beta^{-1}
$$

Thus we have

$$
\left\langle f_{i},\left[\beta x_{j}\right]_{x}\right\rangle=\left\langle f_{i}, \beta e_{j} \beta^{-1}\right\rangle=\left\langle f_{i}, \beta e_{j} \tau(\beta)^{-1}\right\rangle=\left\langle f_{i}, \beta e_{j}\right\rangle \tau(\beta)^{-1}=t_{i} r_{i j} \tau(\beta)^{-1}
$$

Since $t_{i}$ and $\tau(\beta)$ are not zero divisors elements of $\mathcal{M}$ we see $r_{i j}=0$ if and only if

$$
\left\langle f_{i},\left[\beta x_{j}\right]_{x}\right\rangle=0
$$

Now $f_{i}$ itself is the homology class of an arc $y_{i}$ based at $y$ whose homotopy class is $\mathbf{y}_{\mathbf{i}}$. This is an arc which encircles only $q_{i}$ but it goes around to the left, and comes in from the top, and is oriented clockwise. Also the arc $x_{j}$ is based at $x$ and encircles $q_{j}$ anticlockwise. The reason for this difference in orientation 
is that the basis elements of $\oplus \mathcal{M} f_{i}$ (resp. $\oplus e_{i} \mathcal{M}$ ) are the $f_{i}^{\prime} s$ (resp. $e_{i}^{\prime} s$ ), which correspond to clockwise (resp anticlockwise) loops based at $y$ (resp. $x$ ).

Now since $f_{i}=\left[y_{i}\right]_{y}$ we see from Theorem 1 that (23) is equivalent to the assertion that $y_{i}$ can be homotoped off $\beta\left(x_{j}\right)$.

Since $\beta\left(x_{j}\right)$ does not now meet $y_{i}$ then the word $\beta\left(\mathbf{x}_{\mathbf{j}}\right)$ in the free group does not involve the letter $\mathbf{x}_{\mathbf{i}}$. That means the braid $\beta$ can be factorized bc so that strand $j$ at the bottom only has overcrossings in $c$ and strand $i$ at the top only undercrossings in $b$. To see this, consider $\beta$ applied to straight arcs from a basepoint $r$ to the $q_{s}$. The motions of the $q_{s}$ are approximated by letting the $q_{s}$ move out along these arcs from the basepoint to their final positions. Now parametrize the $j$ 'th arc slower than the others, so in the early part of the braid $q_{j}$ is in front of all other points (nearer the basepoint). This gives the $c$ part of

the braid. And for a second interval of time only $q_{j}$ moves and this never moves behind $q_{i}$. This gives the $b$ part of the braid. $\|$

Now we can give the corollary describing how to recognize exchange moves and reducing moves by looking at a single matrix entry. Of course this is much weaker than Theorems 2 and 3 because there we find reducing moves and exchange moves anywhere in the conjugacy class, but we include this result as it was the first one which we noticed.

Corollary 1 1. The matrix entry $r_{n, n-1}$ is zero if and only if $\beta=P \sigma_{n-1}^{-1} Q \sigma_{n-1}$ for some $P, Q \in \mathbf{B}_{n-1}$. In this case $\beta$ admits an exchange move which replaces it by $Q \sigma_{n-1}^{-1} P \sigma_{n-1}$.

2. The matrix entry $r_{n, n}$ is zero if and only if $\beta=P \sigma_{n-1}^{-1} Q$ for some $P, Q \in \mathbf{B}_{n-1}$. In this case $\beta$ admits a reduction move replacing it by $Q P$.

Proof: Apply the lemma first in the special case $j=n-1$ and $i=n$. This shows $\beta$ is of the form $P \sigma_{n-1}^{-1} Q \sigma_{n-1}$. If $i$ and $j$ are both equal to $n$ then $\beta=P \sigma_{n-1}^{-1} Q$. , and this proves the corollary. $\|$

But Theorems 2 and 3 show there is nothing special about the loops $\gamma$ and $\delta$ in the proof of the lemma. Any pair of disjoint simple loops would give rise to an exchange if the transform of one (based at $x$ ) doesn't meet the other (based at $y$ ). Thus, what we have accomplished is to generalize a sort of naive notion about matrix entries into a result giving effective obstructions for reduction or exchange.

\section{Questions, Comments and Conjectures}

We end this paper by discussing the things we have not been able to do, mentioning some conjectures and open problems.

1. A conjecture: Theorem प(e) gives a necessary condition for a homology class $[w]_{x} \in H_{1}\left(P_{n}, x, \mathcal{M}\right)$ to be represented by a simple arc. We conjecture that there is a series of finite-dimensional representations $\tau: B_{1, n} \rightarrow \mathcal{M}$ satisfying the conclusions (a) through (f) of the Theorem 1, for which this condition is sufficient as well necessary. It is even possible that the condition is sufficient in the special case which we discussed in Section 2.3 
2. An important question: Theorem 1 imposes necessary conditions which an element $\beta \in G l_{n}(\mathcal{M})$ must satisfy if $\beta$ is to represent a braid. Namely, for any homology class $v \in \oplus e_{i} \mathcal{M}$ we define $\beta v$ by the ordinary action, and we can define $v^{\star} \beta$ by the rule

$$
v^{\star} \beta=\left(\beta^{-1} v\right)^{\star} .
$$

Then in order to represent a braid, $\beta$ must satisfy $\left\langle v^{\star} \beta, w\right\rangle=\left\langle v^{\star}, \beta w\right\rangle$ for all $v, w$. Secondly, the special homology class $u=\left[x_{1} x_{2} \ldots x_{n}\right]_{x}$ must satisfy $\beta u=u \tau(\beta)$. It would be interesting to know what other elementary algebraic conditions characterize the set of matrices which represent braids. The more completely this question can be answered, the closer one comes to finding an algebraic description of the set of simple homology classes, which in view of theorems 2 and 3 is all that is needed to immediately recognize whether a braid admits an exchange or reducing move.

3. A comment: Since $\left\langle v^{*}, \beta v \beta^{-1}\right\rangle=\left\langle v^{*}, \beta v\right\rangle \beta^{-1}$, the condition which is given for recognizing a reducible braid, in Theorem 2 is equivalent to the apparently simpler condition $\left\langle v^{*}, \beta v\right\rangle=0$. However the more complicated condition has the advantage that the homology class $\beta v \beta^{-1}$ is simple if and only if $v$ is, and the theorem is an assertion about simple homology classes, so we prefer the version we have stated.

4. A question: One may construct a representation $\tau_{0}: \mathbf{B}_{1, n} \rightarrow \mathcal{M}$ which describes the action of $B_{1, n}$ on the Laurent polynomial ring $R=\mathbb{Z}\left[T_{1}^{ \pm 1}, \ldots, T_{n}^{ \pm 1}\right]$. The generator $\sigma_{i}$ acts by permuting $T_{i}$ and $T_{i+1}$ while the generator $\mathbf{x}_{i}$ acts by multiplication by $T_{i}$ and $\mathcal{M}$ is taken to be the endomorphism ring of $R$ as a module over the subring of symmetric polynomials. The matrix entries $r_{i j}$ of the $\tau_{0}^{+}$representation are elements of $R$ composed with substitutions which permute the variables. Does the equation $r_{n, n}=0$ characterise braids of the special reducible form $P \sigma_{n-1}^{-1} Q$ of section 4 ?

5. A comment: When the $\mathcal{M}$ are matrix rings $\mathcal{M}=\operatorname{End}(V)$ the bilinear form of Theorem 1 can be defined on the actual representation $V \oplus V \oplus \ldots \oplus V$ rather than a sum of matrix rings. Let $\epsilon \in \mathcal{M}$ such that $V=\mathcal{M} \epsilon$ occurs as a principal left ideal. Then the bilinear form on $\oplus \epsilon^{*} \mathcal{M} f_{i} \quad \times \oplus e_{i} \mathcal{M} \epsilon \quad$ is defined by the formula $\left\langle\epsilon^{*} v, w \epsilon\right\rangle=\epsilon^{*}\langle v, w\rangle \epsilon$.

6. A comment: The bilinear form we have constructed in this paper is symmetric in the sense that $\langle v, w\rangle=\left\langle w^{\star}, v^{\star}\right\rangle$.

7. A comment: In our survey paper [9], joint with Darren Long, we stated without proof the following result generalizing both [25] and [21]:

Lemma 7.1 ( Lemma 2.6 of [9]) Suppose the representations $\tau$ of $B_{n}$ are not all faithful. Then for all but finitely many values of $n$ the representation $\tau^{+}$of $\mathbf{B}_{n}$ is faithful if and only if the monodromy representation $\mathbf{F}_{n} \subset \mathbf{B}_{1, n} \stackrel{\tau}{\rightarrow} \mathcal{M}$ defines an effective intersection theory for arcs in $P_{n}$.

D. Long has proven the lemma for nonabelian monodromy and cap products [20. In Theorem 1 we establish the Lemma, interpreting the 
intersection form as intersections of two arcs in $P_{n}$ based at two different points $x, y$ on the braid axis. The non-degeneracy of the intersection pairing is similar to Deligne's non-degenerate form on cohomology described in [20].

8. A comment: When $\mathcal{M}$ has an anti-involution $\star$ compatible with $\tau$, as required by the hypotheses of Theorem 1 , it is also true in turn that the ring $\operatorname{Mat}_{n}(\mathcal{M})$ of matrices over $\mathcal{M}$ has anti-involution compatible with $\tau^{+}$. This is defined such that a matrix $B$ is sent to the matrix $B^{\prime}$ such that $\left(B f_{i}^{\star}\right)^{\star}=f_{i} B^{\prime}$ for all $i$. The process of passing from $\tau$ to $\tau^{+}$may then be iterated, obtaining $\tau^{++}$and so-on, and the conclusions of Theorem 1 are retained at each stage. Although one now knows that the question of faithfulness is resolved at the first stage, our conjecture 1 above allows the possibility of a further 'augmentation' before it may be expected to be true.

9. Our final remarks deal with relationship between Theorem 1 part (f), and Bigelow's 'Key Lemma' (Lemma 3.1 of [2]). Our theorem applies to any representation $\tau$ for which $\tau^{+}$is faithful, whereas Bigelow's original proof involves counting monomial degrees in one particular representation. Our theorem in some sense generalizes Bigelow's Key Lemma to the cases where we specialize the parameters $q$ and/or $T$ to values for which faithfulness is known. For example, by [18] we could specialize $q$ to any real number between 0 and 1 . It might be worthwhile to prove this implication (i.e. that Krammer's work implies a generalization of Bigelow's Key Lemma) more precisely.

Continuing: we interpret the intersection form as intersections of two loops on the plane $P$ based at distinct points. The same formula generalizes to any pair of 'noodles.' For the geometric applications, in the special case $\mathcal{M}$ is taken to be the Magnus representation, it would suffice to directly use Bigelow's lemma in place of our part (f) by replacing $n$ by $n+1$ and approximating one of our arcs by a noodle and the other by a fork leading to the extra point $q_{n+1}$.

Finally, the homology classes we consider are the ordinary (first) homology classes of our arcs, but with coefficients in a noncommutative ring. In this theory the self-intersections of an arc are interesting.

\section{References}

[1] S. Bigelow, The Burau representation of $B_{5}$ is not faithful, Geometry and Topology 3 (1999), 397-404.

[2] S. Bigelow, Braid groups are linear, J. AMS 14(2001) 471-486

[3] Birman,J.S. and Menasco, W.: Studying Links Via Closed Braids IV: Closed Braid Representatives of Split and Composite Links, Inventiones Math, 102 Fasc. 1 (1990), 115-139. 
[4] Birman,J.S. and Menaso, W.: Studying Links Via Closed Braids V: Closed Braid Representatives of the Unlink, Trans AMS, 329 No. 2 (1992) pp. 585-606.

[5] Birman,J.S. and Menaso, W.: Studying Links Via Closed Braids VI:A Non-Finiteness Theorem, Pacific J. Math., 156, No. 2, 1992, p. 265-285.

[6] J. Birman, Braids, Links and Mapping Class Groups Annals of Mathematics Studies 82, Princeton University Press 1974

[7] J. Birman and M. Hirsch, A new algorithm for recognizing the unknot, Geometry and Topology 2 (1998) 175-220

[8] J. Birman, A. Lubotzky and J. McCarthy, Abelian and solvable subgroups of the mapping class group, Duke Math. J. 50 No. 4 (1983), 1107-1120.

[9] J. Birman, D. Long, J. Moody, Finite-dimensional representations of Artin's braid group, The Mathematical Legacy of Wilhelm Magnus, Contemporary Math 169 (1994), 123-132.

[10] W. Burau, Uber Zopfgruppen und gleichsinning verdrillte Verkettunger, Abh. Math. Sem. Hanischen Univ. 11 (1936) 171-178.

[11] A. Fathi, F. Laudenbach and V. Peonaru, Traveaux de Thurston sur les surfaces Asterisque 66-67 (1979), Societe Mathematique de France.

[12] J. Fehrenbach, "Quelques aspects géométriques et dynamiques du mapping class group" January 1998, PhD thesis, Université de Nice.

[13] T. Fiedler, A small state sum for knots, Topology 32 (1993), 281-294.

[14] B. J. Gassner, On braid groups, Abh, Math, Sem. Hamburg Univ. 25 (1961), 19-22.

[15] N. Ivanov, Subgroups of the Teichmuller Modular Group, American Math Soc. Translations of Mathematical Monographs 115

[16] V.F.R. Jones, Hecke algebra representations of braid groups and link polynomials, Annals of Math. 126 (1987), 335-388.

[17] D. Krammer, The braid group $B_{4}$ is linear, Inventiones 142, 451-48 
[18] D. Krammer, Braid groups are linear, Annals of Mathematics, to appear.

[19] R. J. Lawrence, Homological representations of the Hecke algebra, Comm. Math. Physics 135 (1990), No. 1,141-191.

[20] D. Long, Constructing representations of braid groups, Comm Math Anal Geom 2(1994) 217-238

[21] D.Long and M. Patton, The Burau representation is not faithful for $n \geq$ 6, Topology 32 (1993), no. 2, 439-447.

[22] J. McCarthy, A Tits alternative for subgroups of mapping class groups, Trans AMS 291 (1985) 583-612.

[23] J. McCool, On reducible braids, in "Word Problems II", editors Adian, Boone and Higman, North-Holland (1980), 261-295.

[24] W. W. Menasco, On iterated torus knots and transversal knots, Geometry and Topology 5 (2001), 651-682.

[25] J. Moody, The faithfulness question for the Burau representation, AMS Proc 119, 1993, 671-679

[26] H. Morton, An irreducible 4-braid with unknotted closure, Math. Proc. Cambridge Phil. Soc., 93 (1983), 259-261. 